May 1996

CBPF-NF-021/96

TAUP 233896/96

\title{
DIFFRACTIVE LEPTOPRODUCTION OF SMALL MASSES IN QCD
}

\author{
E R R O L G O T S M A N ${ }^{1)}$ \\ School of Physics and Astronomy \\ Raymond and Beverly Sackler Faculty of Exact Science \\ Tel Aviv University, Tel Aviv, 69978, ISRAEL \\ E U G E N E L E V I N ${ }^{2)}$ \\ LAFEX, Centro Brasileiro de Pesquisas Físicas (CNPq) \\ Rua Dr. Xavier Sigaud 150, 22290 - 180 Rio de Janeiro, RJ, BRASIL \\ and \\ Theory Department, Petersburg Nuclear Physics Institute \\ 188350, Gatchina, St. Petersburg, RUSSIA \\ U R I M A O R ${ }^{3)}$ \\ School of Physics and Astronomy \\ Raymond and Beverly Sackler Faculty of Exact Science \\ Tel Aviv University, Tel Aviv, 69978, ISRAEL
}

\footnotetext{
1) Email: gotsman@post.tau.ac.il .

2) Email: levin@fnalv.fnal.gov .

3) Email: maor@ccsg.tau.ac.il .
} 


\begin{abstract}
In this paper we consider the process of diffraction dissociation in deep inelastic scattering producing a small mass. This process is analyzed by the calculation of $\bar{q} q$ and $\bar{q} q G$ production. We show that the small distance contributions $\left(r_{\perp} \propto 1 / Q\right)$ to the longitudinal polarised virtual photon dominate the diffractive channels. Formulae for the cross section using the gluon density are written within the framework of perturbative QCD. We show that the production of small masses by a transverse polarised photon is concentrated at moderate values of $r_{\perp} \sim 1 \mathrm{GeV}^{-1}$, where the pQCD approach is valid. It is shown that only $\bar{q} q$ pair production contributes to diffraction dissociation with $\beta>0.4$ and the possibility to extract the values of the gluon density from the measurements in this kinematic region is discussed. Shadowing corrections are assessed for both longitudinal and transverse polarised photons and estimates of the different damping factors are given. The relation between diffractive production and the corrections to $F_{2}$ is alluded to. The evolution of the diffractive structure function is studied and a solution to the diffractive evolution equations is proposed.
\end{abstract}




\section{Introduction}

Over the last decade, starting with the paper of Bartels and Loewe [1], diffractive processes in deep inelastic scattering (DIS) have attracted a great deal of attention. The reason is that these processes can be calculated within the framework of perturbative QCD (pQCD) 2] and their calculated cross sections turn out to be proportional to the square of the gluon density $x_{P} G\left(Q^{2}, x_{P}\right) . Q^{2}$ is the virtuality of the photon and $x_{P}=\frac{Q^{2}+M^{2}}{s}$, where $s$ is the c.m. energy squared of the reaction. $M$ is the mass of the diffractively produced hadronic system. The recent renewed interest in these channels was triggered by Ryskin[3] and Brodsky et.al. [4], who proved that vector meson electro production can be calculated in the leading log approximation of pQCD, while the non perturbative effects, originating from large distances, can be factored out in terms of the light-cone $\bar{q} q$ wave function of the produced vector meson.

The goal of this paper is to study single diffraction dissociation (DD) production of a small mass hadronic system. We consider a small mass as the hadronized product of either a $\bar{q} q$ pair or a $\bar{q} q G$ system in the final state of the DD process. We will show that: 1. This process describes a significant part of the experimental DD cross section.

2. The small distance contribution $\left(r_{\perp} \propto 1 / Q\right)$ dominates the longitudinal polarised virtual photon which induces DD. This justifies the use of a pQCD approach to calculate the relevant cross section.

3. The production of small masses by a transverse polarised photon are concentrated at moderate values of $r_{\perp}^{2} \approx 1 \mathrm{GeV}^{-2}$. This encourages us to believe, that our estimates which are obtained in pQCD, could be responsible for a considerable part of the DD cross section.

4. Shadowing (or screening) corrections (SC) are not negligble in the region of low $x_{P}$ and should be taken into account.

The plan of our paper is as follows: In the next section we calculate the process of DD by longitudinal and transverse polarized photons into a $\bar{q} q$ pair as well as through $\bar{q} q G$. This is done in the $r_{\perp}$-representation, which provides a natural frame to assess at what distances these processes are viable. It is also a convenient representation to calculate SC. Most of the formulae which we obtain for transverse polarised photons have been previously derived in the momentum representation, see Refs. 50 60 [7]. In section 3 we extend the formalism suggested by Levin and Ryskin[8] and Mueller[9], to the case of a penetration of a $\bar{q} q$ pair with a definite value of $r_{\perp}$ through the proton, as well as through the nucleus. We will show that for the case of a diffractive production of small masses by

* Mark Wüsthoff has done the calculations for a longidudinal polarized photon in his $\mathrm{PhD}$ thesis and presented the results in the 1995 Durham workshop. 
longitudinal polarised photons, we obtain an elegant closed expression for the damping factor [10] defined by

$$
D^{2}=\left.\frac{\frac{d \sigma\left(\gamma^{*}+p \rightarrow X(M)+p\right)}{d t}[\text { with } S C]}{\frac{d \sigma\left(\gamma^{*}+p \rightarrow X(M)+p\right)}{d t}[\text { without } S C]}\right|_{t=0}
$$

where $t=-q_{\perp}^{2}$. Processes at large transverse distances do not enter the above formula, allowing us to calculate the SC within the framework of pQCD. We extend our formalism also to DD by a transverse polarized photon. We discuss the large distance contribution in this case and show that the SC lead to a decrease of the typical distances that are responsible for DD. In section 4 we compare the pQCD predictions with the experimental data available from HERA[11] [12] (see also Refs. [13] [14]). We show that pQCD is able to account for a considerable part of the observed DD cross section, and discuss which distances contribute to the different modes present in the final state of the produced system. In all our calculations we use the GRV parametrization[15] for the gluon density, which is the main input ingredient in our work. The GRV parametrization which starts from a low value of the photon virtuality, allows us to study the value of typical distances in the DD process. In the fifth section we discuss the evolution equations for the DD processes. A summary of our results and a discussion are presented in the conclusions.

\section{The diffractive production of $\bar{q} q$ and $\bar{q} q G$ systems without shadowing corrections}

\section{$2.1 \quad$ Notation}

We list below the notation used in this paper (see Fig.1).

1. $Q^{2}$ is the virtuality of the photon in DIS.

2. $x_{P}=\frac{Q^{2}+M^{2}}{s}$, where $s$ denotes the square of the c.m. energy and $M$ is the produced diffractive mass. Bjorken's scaling variable is given by $x_{B}=\frac{Q^{2}}{s}$.

3. $\beta=\frac{Q^{2}}{Q^{2}+M^{2}}=\frac{x_{B}}{x_{P}}$.

4. $a^{2}=z(1-z) Q^{2}+m_{Q}^{2}$, where $z$ is the fraction of the photon energy that is carried away by a quark with a mass $m_{Q}$.

5. $\quad \vec{k}_{\perp}$ denotes the transverse momentum of the quark, and $\vec{r}_{\perp}$ the transverse distance between the quark and the antiquark.

6. $\vec{b}_{\perp}$ is the impact parameter of the reaction and is the conjugate variable to $\vec{q}_{\perp}$, the momentum transfer from the incoming proton to the recoiled proton. Note that $t=-q_{\perp}^{2}$. 
7. $\vec{l}_{i \perp}$ denotes the transverse momentum of gluons, which are attached to the quarkantiquark pair (see Fig.1).

8. We use the evolution equations for the parton densities in moment space. For any function $f(x)$, we define the moment $f(\omega)$ as

$$
f(\omega)=\int_{0}^{1} d x x^{\omega} f(x)
$$

Note that the moment variable $\omega$ is chosen so that the $\omega=0$ moment measures the number of partons and the $\omega=1$ moment measures their momentum. An alternative moment variable $\mathrm{N}$, defined such that $\mathrm{N}=\omega+1$, is often found in the literature.

The $x$ - distribution can be reconstructed by considering the inverse Mellin transform. For example, for the gluon density it reads

$$
x G\left(Q^{2}, x\right)=\frac{1}{2 \pi i} \int_{C} d \omega x^{-\omega} g\left(Q^{2}, \omega\right)
$$

The contour of integration $\mathrm{C}$ is taken to the right of all singularities.

For the solution of the DGLAP equations [16], as well as the BFKL equation [17], one has the following form

$$
g\left(Q^{2}, \omega\right)=g(\omega) e^{\gamma(\omega) \ln Q^{2}}
$$

where $\gamma(\omega)$ denotes the anomalous dimension which, in the leading $\log \frac{1}{x}$ approximation of pQCD, is a function of $\frac{\alpha_{\mathrm{S}}}{\omega}$ and can be presented as 18

$$
\gamma(\omega)=\frac{\alpha_{\mathrm{S}} N_{C}}{\pi} \frac{1}{\omega}+\frac{2 \alpha_{\mathrm{S}}^{4} N_{C}^{4} \zeta(3)}{\pi^{4}} \frac{1}{\omega^{4}}+O\left(\frac{\alpha_{\mathrm{S}}^{5}}{\omega^{5}}\right)
$$

9. Our amplitude is normalized so that

$$
\frac{d \sigma}{d t}=\pi|f(s, t)|^{2}
$$

and the optical theorem can be written

$$
\sigma_{\text {tot }}=4 \pi \operatorname{Im} f(s, 0)
$$

The scattering amplitude in $b_{\perp}$-space is defined by

$$
a\left(s, b_{\perp}\right)=\frac{1}{2 \pi} \int d^{2} q_{\perp} e^{-i \vec{q}_{\perp} \cdot \vec{b}_{\perp}} f\left(s, t=-q_{\perp}^{2}\right)
$$

In this representation

$$
\sigma_{\text {tot }}=2 \int d^{2} b_{\perp} \operatorname{Im} a\left(s, b_{\perp}\right)
$$

and

$$
\sigma_{e l}=\int d^{2} b_{\perp}\left|a\left(s, b_{\perp}\right)\right|^{2}
$$

In what follows we use the notation and normalization of Brodsky et.al. [4]. 


\subsection{The cross section for diffractive dissociation}

The cross section for the diffractive process (see Fig.1)

$$
\gamma^{*}\left(Q^{2}, x_{B}\right)+p \rightarrow X\left(M^{2}\right)+p
$$

can be written in the form

$$
\begin{gathered}
x_{P} \frac{d \sigma}{d x_{P} d t}=\frac{3 \alpha_{e m}}{8(2 \pi)^{2}} \sum_{1}^{N_{F}} Z_{F}^{2} \sum_{\lambda_{1} \lambda_{2}} \int_{0}^{1} d z \int \frac{d k_{\perp}^{2}}{(2 \pi)^{2}} \\
\left|f\left(\gamma^{*}+p \rightarrow q\left(z, k_{\perp}\right)+\bar{q}\left(1-z,-k_{\perp}+q_{\perp}\right)+p\right)\right|^{2}\left(M^{2}+Q^{2}\right) \delta\left(M^{2}-\frac{k_{\perp}^{2}}{z(1-z)}\right)
\end{gathered}
$$

We have assumed in eq.(7) that the small masses are produced by the dissociation of a $\bar{q} q$ system. We will consider later, the corrections resulting from the emission of gluons. In eq. (7), $\lambda_{i}$ denotes the polarisation of the quark, $N_{F}$ the number of quark flavours and $Z_{F}$ the fraction of the electron charge carried by the quark with a flavour $F$.

Integrating over $z$ and summing over polarizations, we reduce the above equation to the form

$$
x_{P} \frac{d \sigma}{d x_{P} d t}=\frac{3 \alpha_{e m}}{(2 \pi)^{3}} \sum_{1}^{N_{F}} Z_{F}^{2} N_{\lambda} \int|f|^{2} \frac{d^{2} k_{\perp}}{1-\beta} \frac{k_{\perp}^{2}}{M^{2}} \frac{2}{\sqrt{1-\frac{4 k_{\perp}^{2}}{M^{2}}}}
$$

where $z(1-z)=\frac{k_{\perp}^{2}}{M^{2}}$ and $a=\sqrt{z(1-z) Q^{2}+m_{Q}^{2}}=Q \frac{k_{\perp}}{M}$ for massless quarks. The region of integration with respect to $k_{\perp}$ is defined $k_{\perp}^{2}<\frac{M^{2}}{4}$. The factor $N_{\lambda}$ depends on the initial polarization of the photon. In the case of longitudital polarization $N_{\lambda}=4$. For transverse polarization $N_{\lambda}=z^{2}+(1-z)^{2}$. We follow Refs. [5] 9] regarding normalization, and the definition of the photon wave function. The main task before us is the calculation of the amplitude $f$.

\subsection{The amplitude in the $r_{\perp}$ representation}

This approach was first formulated in Ref.[8] and has been carefully developed in Ref.[9]. During it's time of passage through the target, the distance $r_{\perp}$ between a quark and an antiquark can vary by an amount $\Delta r_{\perp} \propto R \frac{k_{\perp}}{E}$. E denotes the energy of the pair and $R$ the size of the target (see Fig.1). The quark's transverse momentum is $k_{\perp} \propto \frac{1}{r_{\perp}}$. Therefore

$$
\Delta r_{\perp} \propto R \frac{k_{\perp}}{E} \ll r_{\perp}
$$


holds if

$$
r_{\perp}^{2} s \gg 2 m R
$$

where $s=2 m E$.

The above condition can be rewritten in terms of $x_{P}$

$$
x_{P} \ll \frac{2}{(1-\beta) m R}
$$

This means that at small values of $x_{P}$, the transverse distance between the quark and the antiquark is a good degree of freedom 8] [19], and the interaction of a virtual photon with the target can be written in the form

$$
f=\int \frac{d^{2} r_{\perp}}{2 \pi} \Psi^{*}\left(r_{\perp}, k_{\perp}\right) \sigma\left(r_{\perp}, q_{\perp}^{2}\right) \Psi^{\gamma^{*}}\left(Q^{2}, r_{\perp}, k_{\perp}\right)
$$

$\Psi$ denotes the wave function of the produced $\bar{q} q$ pair which is equal to $e^{i \vec{r}_{\perp} \cdot \vec{k}_{\perp}}$. After integrating over the azimuthal angle we have $J_{0}\left(k_{\perp} r_{\perp}\right)$ and $J_{1}\left(k_{\perp} r_{\perp}\right)$ for the longitudinal and transverse polarised photon induced reactions respectively. $\Psi^{\gamma^{*}}$ denotes the wave function of the longitudinally ( $\mathrm{L}$ ) or transverse ( $\mathrm{T}$ ) polarized photon. These have been given in Refs. [5][9] and are equal to

$$
\Psi_{L}^{\gamma^{*}}\left(Q^{2}, r_{\perp}, z\right)=Q z(1-z) K_{0}\left(a r_{\perp}\right)=Q \frac{k_{\perp}^{2}}{M^{2}} K_{0}\left(a r_{\perp}\right)
$$

and

$$
\Psi_{T}^{\gamma^{*}}\left(Q^{2}, r_{\perp}, k_{\perp}\right)=i a K_{1}\left(a r_{\perp}\right) \frac{\overrightarrow{r_{\perp}}}{r_{\perp}}
$$

$\sigma\left(r_{\perp}, q_{\perp}^{2}\right)$ denotes the cross section of the $\bar{q} q$ pair with a transverse separation $r_{\perp}$, which scatters with transverse momentum $q_{\perp}$. We will discuss it in detail below, considering initially the case where $q_{\perp}=0$.

\section{$2.4 \sigma\left(r_{\perp}, q_{\perp}^{2}\right)$ at $q_{\perp}=\mathbf{0}$}

The form for $\sigma\left(r_{\perp}, q_{\perp}^{2}\right)$ at $q_{\perp}$ has been given in Ref. [8] (see eq. (8) of this paper). $\sigma$ can be expressed in terms of the unintegrated parton density $\phi$, first introduced in the BFKL papers 17] and widely used in Ref. 20]. This function is clearly related to the gluon density and can be calculated using the equation

$$
\alpha_{\mathrm{S}}\left(Q^{2}\right) x G\left(Q^{2}, x\right)=\int^{Q^{2}} d l_{\perp}^{2} \alpha_{\mathrm{S}}\left(l_{\perp}^{2}\right) \phi\left(l_{\perp}^{2}, x\right)
$$


Using the above equation we obtain the result of Ref. [8], which reads

$$
\sigma\left(r_{\perp}, q_{\perp}^{2}\right)=\frac{16 C_{F}}{N_{C}^{2}-1} \pi^{2} \int \phi\left(l_{\perp}^{2}, x\right)\left(1-e^{i \vec{l}_{\perp} \cdot \vec{r}_{\perp}}\right) \frac{\alpha_{\mathrm{S}}\left(l_{\perp}^{2}\right)}{2 \pi} \frac{d^{2} l_{\perp}}{l_{\perp}^{2}}
$$

where $\phi=\frac{\partial x G\left(Q^{2}, x\right)}{\partial Q^{2}}$.

We calculate this integral using eqs.(3) and (4), and integrate over the azimuthal angle. Introducing a new variable $\xi=r_{\perp} l_{\perp}$ we can reduce the integral to the form

$$
\sigma\left(r_{\perp}, q_{\perp}^{2}\right)=\frac{16 C_{F} \alpha_{\mathrm{S}}}{N_{C}^{2}-1} \pi^{2} \int_{C} \frac{d \omega}{2 \pi i} g(\omega) \gamma(\omega)\left(r_{\perp}^{2}\right)^{1-\gamma(\omega)} \int_{0}^{\infty} d \xi \frac{1-J_{0}(\xi)}{(\xi)^{3-2 \gamma(\omega)}}
$$

preforming the integration over $\mathrm{d} \xi$ (see Ref. 21 11.4.18) we have

$$
\sigma\left(r_{\perp}, q_{\perp}^{2}\right)=\frac{8 C_{F} \alpha_{\mathrm{S}}}{N_{C}^{2}-1} \pi^{2} \int_{C} \frac{d \omega}{2 \pi i} g(\omega) \gamma(\omega)\left(\frac{r_{\perp}^{2}}{4}\right)^{1-\gamma(\omega)} \frac{\Gamma(\gamma(\omega)) \Gamma(1-\gamma(\omega))}{(\Gamma(2-\gamma(\omega)))^{2}}
$$

In the double leading log approximation of pQCD, $\gamma(\omega) \ll 1$, and we obtain the following cross section for $N_{C}=3$

$$
\sigma\left(r_{\perp}, q_{\perp}^{2}\right)=\frac{\alpha_{\mathrm{S}}\left(\frac{4}{r_{\perp}^{2}}\right)}{3} \pi^{2} r_{\perp}^{2}\left(x G^{D G L A P}\left(\frac{4}{r_{\perp}^{2}}, x\right)\right)^{2}
$$

This result agrees with the expression for the cross section value given in Refs.[22] and [23] provided we neglect the factor 4 in the argument of the gluon density. We have checked that eq. (2.16) of Ref. 四 also leads to the same result, unlike the value for $\sigma$ quoted in the paper (see eq. (2.20) in Ref. 四).

\subsection{The amplitude for small mass production at $q_{\perp}=0$ induced by a longitudinal photon}

In this section we calculate the amplitude for $\bar{q} q$ production with a mass $M$. Substituting eq. (13), in our expression (12) for $f$, yields

$$
\begin{aligned}
& f=\int r_{\perp} d r_{\perp} \frac{Q k_{\perp}^{2}}{M^{2}} K_{0}\left(\frac{Q}{M} k_{\perp} r_{\perp}\right) \frac{8 C_{F} \alpha_{S}}{N_{C}^{2}-1} \pi^{2} \int_{C} \frac{d \omega}{2 \pi i} \\
& g(\omega) \gamma(\omega)\left(\frac{r_{\perp}^{2}}{4}\right)^{1-\gamma(\omega)} \frac{\Gamma(\gamma(\omega)) \Gamma(1-\gamma(\omega))}{(\Gamma(2-\gamma(\omega)))^{2}} J_{0}\left(k_{\perp} r_{\perp}\right)
\end{aligned}
$$


Making use of the well known properties of the modified Bessel functions we can perform the integration. The final general result is

$$
f=\frac{Q k_{\perp}^{2}}{M^{2}} \frac{8 C_{F} \alpha_{\mathrm{S}}}{N_{C}^{2}-1} \pi^{2} \int_{C} \frac{d \omega}{2 \pi i} g(\omega) \frac{1}{a^{4}}\left(\frac{a^{2}}{\beta}\right)^{\gamma(\omega)}
$$

$\Gamma(1+\gamma(\omega)) \Gamma(1-\gamma(\omega)) \Gamma(2-\gamma(\omega)) \Gamma(2-\gamma(\omega)) \beta^{3}{ }_{1} F_{1}(2-\gamma(\omega),-1+\gamma(\omega), 1,1-\beta)$ where $a=Q \frac{k_{\perp}}{M}$.

In the case of the DGLAP approach we can simplify eq. (21) as $\gamma(\omega) \ll 1$. Neglecting $\gamma(\omega)$ with respect to 1 , and using eq. (3) we get

$$
f=-\pi^{2} \frac{8 C_{F} \alpha_{\mathrm{S}}}{N_{C}^{2}-1} \frac{Q \frac{k_{\perp}^{2}}{M^{2}}}{\left(Q^{2} \frac{k_{\perp}^{2}}{M^{2}}\right)^{2}} \beta^{2} x_{P} G\left(\frac{k_{\perp}^{2}}{(1-\beta)}, x_{P}\right)(1-2 \beta)
$$

It is interesting to note that the argument of $x_{P} G$ is $k_{\perp}^{2} /(1-\beta)$, which means that small distances of the order of $r_{\perp}^{2} \propto(1-\beta) / k_{\perp}^{2}$, contribute to small mass diffractive production, especially at $\beta \rightarrow 1$.

\subsection{DD cross section induced by a longitudinal photon at $t=0$}

Subtituting the amplitude in eq. (8) for the cross section, we have for three flavours and three colours

$$
\begin{gathered}
\left.x_{P} \frac{d \sigma}{d x_{P} d t}\right|_{t=0}=\frac{4 \pi^{2}}{3} \alpha_{e m} \alpha_{\mathrm{S}}^{2} \sum_{F} Z_{F}^{2} \frac{1}{Q^{4}} \beta^{3}(1-2 \beta)^{2} \\
\int_{Q_{0}^{2}}^{\frac{M^{2}}{4}} \frac{d k_{\perp}^{2}}{k_{\perp}^{2}}\left(x_{P} G\left(\frac{k_{\perp}^{2}}{(1-\beta)}, x_{P}\right)\right)^{2} \frac{1}{\sqrt{1-\frac{4 k_{\perp}^{2}}{M^{2}}}}
\end{gathered}
$$

One can conclude from eq. (23) that only small distance processes contribute to the cross section. This fact manifests itself in the log integration with respect to $k_{\perp}^{2}$. Even in the double log approximation of pQCD, this integral converges and can be rewritten in the form

$$
\begin{gathered}
\left.x_{P} \frac{d \sigma}{d x_{P} d t}\right|_{t=0}=\frac{4 \pi^{3}}{3 N_{C}} \alpha_{e m} \alpha_{\mathrm{S}}^{3} \sum_{F} Z_{F}^{2} \frac{1}{Q^{4}} \beta^{3}(1-2 \beta)^{2} \\
\int_{x_{P}}^{1} \frac{d x_{P}^{\prime}}{x_{P}^{\prime}}\left(\frac{\partial\left(x_{P} G\left(\frac{M_{\perp}^{2}}{4(1-\beta)}, x_{P}\right)\right.}{\partial \ln \left(1 / x_{P}^{\prime}\right)}\right)^{2}
\end{gathered}
$$

The above formula gives the result for the case of a two jet production originating from a $\bar{q} q$ pair. 


\subsection{The DD amplitude at $q_{\perp}=0$ for a transverse photon}

Using the formulae given in the previous subsections, and taking into account eqs.(3) and

(4) for the gluon structure function, we obtain the following expression for the amplitude $f:$

$$
\begin{gathered}
f=\int r_{\perp} d r_{\perp} a K_{1}\left(\frac{Q}{M} k_{\perp} r_{\perp}\right) \frac{8 C_{F} \alpha_{\mathrm{S}}}{N_{C}^{2}-1} \pi^{2} \int_{C} \frac{d \omega}{2 \pi i} \\
g(\omega) \gamma(\omega)\left(\frac{r_{\perp}^{2}}{4}\right)^{1-\gamma(\omega)} \frac{\Gamma(\gamma(\omega)) \Gamma(1-\gamma(\omega))}{(\Gamma(2-\gamma(\omega)))^{2}} J_{1}\left(k_{\perp} r_{\perp}\right)
\end{gathered}
$$

Making use of the properties of the modified Bessel functions we perform the integration over $r_{\perp}$ and obtain

$$
\begin{gathered}
f=\frac{8 C_{F} \alpha_{\mathrm{S}}}{N_{C}^{2}-1} \pi^{2} k_{\perp} \int_{C} \frac{d \omega}{2 \pi i} g(\omega) \frac{1}{a^{4}}\left(\frac{a^{2}}{\beta}\right)^{\gamma(\omega)} \\
\frac{\Gamma(1+\gamma(\omega)) \Gamma(1-\gamma(\omega)) \Gamma(2-\gamma(\omega)) \Gamma(3-\gamma(\omega))}{\Gamma(2-g(\omega)) \Gamma(2)} \beta^{3}{ }_{1} F_{1}(3-\gamma(\omega), g(\omega), 2,1-\beta)
\end{gathered}
$$

where $a=Q \frac{k_{\perp}}{M}$.

In the DGLAP approach the above equation can be simplified as $\gamma(\omega) \ll 1$, it reduces to the form

$$
f=\pi^{2} \frac{16 C_{F} \alpha_{\mathrm{S}}}{N_{C}^{2}-1} \frac{k_{\perp}^{2}}{a^{4}} \beta^{3} x_{P} G\left(\frac{k_{\perp}^{2}}{(1-\beta)}, x_{P}\right)
$$

\subsection{DD cross section for a transverse photon into a $\bar{q} q$ pair}

Substituting the amplitude $f$ in the general formula for the cross section (see eq. (8)), we obtain for three flavours

$$
\begin{gathered}
\left.x_{P} \frac{d \sigma}{d x_{P} d t}\right|_{t=0}=\frac{4 \pi^{2}}{3} \alpha_{e m} \alpha_{\mathrm{S}}^{2} \sum Z_{F}^{2} \frac{1}{Q^{2}} \beta^{3}(1-\beta)^{2} \\
\int_{Q_{0}^{2}}^{\frac{M^{2}}{4}} \frac{d k_{\perp}^{2}}{k_{\perp}^{4}}\left(x_{P} G\left(\frac{k_{\perp}^{2}}{(1-\beta)}, x_{P}\right)\right)^{2}\left(1-2 \frac{k_{\perp}^{2}}{M^{2}}\right) \frac{1}{\sqrt{1-\frac{4 k_{\perp}^{2}}{M^{2}}}}
\end{gathered}
$$




\subsection{DD cross section for $\bar{q} q G$ production}

The emission of one additional gluon is shown in the diagrams of Fig.2. One can see two different classes of diagrams which describe gluon emission. In the first, the emitted gluon does not interact with the target as shown in Fig.2a. The general way to take such gluon emission into account, is to use the evolution equations. We shall write down explicitly all formulae for the emission of one gluon at the end of this section. The second class of diagrams describes the process in which the emitted gluon interacts with the target. (see Fig.2b). These diagrams have to be calculated separately so as to provide the initial conditions for the evolution equations [7].

We start with the calculation of the diagrams of Fig.2b. We would like to determine those contributions where the smallness of $\alpha_{\mathrm{S}}$ is compensated by a large logarithm. In other words, we are looking for contributions of the order of $\alpha_{\mathrm{S}} \ln \left(Q^{2} / k^{2}\right)$. Our first observation is that such a term does not exist in the case of a longitudinal polarised photon induced reaction. Indeed, this fact can easily be seen from the general structure of the diagrams of Fig. 2b, namely, they can be written in a general form

$$
\left.x_{P} \frac{d \sigma}{d x_{P} d t}\right|_{t=0}(\text { Fig. } 2 b) \propto \int_{0}^{1} d z \int_{x_{P}}^{1} \frac{d x^{\prime}}{x^{\prime}} \int \frac{d^{2} r_{\perp}}{2 \pi} \Psi^{\gamma^{*}} \sigma\left(r_{\perp}, q_{\perp}^{2}=0, x^{\prime}\right)\left[\Psi^{\gamma^{*}}\right]^{*}
$$

where $\sigma$ is defined by (see eq. (19))

$$
\sigma\left(r_{\perp}, q_{\perp}^{2}\right)=\frac{\alpha_{\mathrm{S}}\left(\frac{4}{r_{\perp}^{2}}\right)}{3} \pi^{2} r_{\perp}^{2}\left(x G\left(\frac{4}{r_{\perp}^{2}}, x\right)\right)^{2}
$$

with $\left[x G\left(r_{\perp}^{2}, x\right)\right]^{2}$ that has been introduced in Ref. 24]. The explicit expression for $(x G)^{2}$ was derived in Ref. [9] and it is equal to

$$
\left(x^{\prime} G\left(r_{\perp}^{2}, x^{\prime}\right)\right)^{2}=\frac{2}{\pi^{2}} \int_{r_{\perp}^{2}}^{\infty} \frac{d^{2} r^{\prime}}{r^{\prime 4}} \int_{0}^{\infty} d b_{\perp}^{2}\left[\sigma^{G G}\right]^{2}
$$

where

$$
\sigma^{G G}\left(r^{\prime}, x^{\prime}\right)=\frac{3 \alpha_{\mathrm{S}}}{4} r^{\prime 2}\left(x^{\prime} G\left(\frac{4}{r^{\prime 2}}, x^{\prime}\right)\right)
$$

Substituting eq. (13) for $\Psi_{L}^{\gamma^{*}}$ in eq. (31), after the integration over $z$ and using the properties of the modified Bessel functions, we obtain

$$
\sigma\left(r_{\perp}, q_{\perp}^{2}\right) \propto \int_{\frac{4}{Q^{2}}}^{\infty} \frac{d r_{\perp}^{2}}{r_{\perp}^{4}} \frac{\alpha_{\mathrm{S}}\left(\frac{4}{r_{\perp}^{2}}\right)}{3} \pi^{2}\left(x G\left(\frac{4}{r_{\perp}^{2}}, x\right)\right)^{2}
$$


Therefore, we have no log contribution from the $r_{\perp}$ integration. However, if we calculate the same integral for the transverse polarised photon we find

$$
\begin{gathered}
\sigma\left(r_{\perp}, q_{\perp}^{2}\right) \propto \int_{\frac{4}{Q^{2}}}^{\infty} \frac{d r_{\perp}^{2}}{r_{\perp}^{2}} \frac{\alpha_{\mathrm{S}}}{3} \pi^{2}\left(x G\left(\frac{4}{r_{\perp}^{2}}, x\right)\right)^{2} \\
\propto \int_{\frac{1}{Q^{2}}}^{\infty} d r^{\prime 2} \ln \left(Q^{2} r^{\prime 2}\right)\left(x G\left(\frac{4}{r_{\perp}^{2}}, x\right)\right)^{2}
\end{gathered}
$$

Finally, going to the $k_{\perp}$ representation, eq. (34) yields the result of Ref. [7]

$$
\begin{gathered}
\left.x_{P} \frac{d \sigma_{T}}{d x_{P} d t}\right|_{t=0}(\text { Fig. } 2 b)=\sum_{F} \frac{4 \pi^{2} \alpha_{e m} Z_{F}^{2}}{Q^{2}} \beta \int_{\beta}^{1} \frac{d z}{z^{4}} \int_{\bar{Q}_{0}^{2}}^{\frac{M^{2}}{4}} \frac{d k^{2}}{k^{4}} \frac{\alpha_{S}^{3} N_{C}^{2}}{32 \pi} \\
\left\{\beta^{2}+(z-\beta)^{2}\right\}(1-z)^{3}(2 z+1)^{2}\left(x_{P} G\left(k^{2}, x_{P}\right)\right)^{2}
\end{gathered}
$$

Taking the integral over $z$ we obtain

$$
\begin{gathered}
\left.x_{P} \frac{d \sigma_{T}}{d x_{P} d t}\right|_{t=0}(\text { Fig. } 2 b)=\sum_{F} \frac{4 \pi^{2} \alpha_{e m} Z_{F}^{2}}{Q^{2}} \int_{\bar{Q}_{0}^{2}}^{\frac{M^{2}}{4}} \frac{d k^{2}}{k^{4}} \frac{N_{C}^{2} \alpha_{S}^{3}}{32 \pi} \ln \frac{M^{2}}{4 k^{2}} \\
\left\{\frac{(1-\beta)}{6}\left[4-31 \beta-63 \beta^{2}+50 \beta^{3}-14 \beta^{4}\right]-\ln \beta\left[1+10 \beta-2 \beta^{2}\right]\right\}\left(x_{P} G\left(x_{P}, k^{2}\right)\right)^{2}
\end{gathered}
$$

We now calculate the diagrams of Fig.2a. For a transverse polarized photon, this has been done in Ref. [7] and the result in our notation is

$$
\begin{gathered}
\left.x_{P} \frac{d \sigma_{T}}{d x_{P} d t}\right|_{t=0}(\text { Fig. } 2 a)=\sum_{F} \frac{4 \pi^{2} \alpha_{e m} Z_{F}^{2}}{Q^{2}} \beta \int_{\beta}^{1} \frac{d z}{z^{5}} \int_{\bar{Q}_{0}^{2}}^{\frac{M^{2}}{4}} \frac{d k^{2}}{k^{4}} \frac{4 \alpha_{\mathrm{S}}^{3}}{3 N_{C} \pi} \ln \frac{M^{2}}{4 k^{2}} \\
\frac{1+z^{2}}{1-z}\left\{\beta^{2}(z-\beta)^{2}-z^{6} \beta^{2}(1-\beta)^{2}\right\}\left(x_{P} G\left(k^{2}, x_{P}\right)\right)^{2}= \\
\sum_{F} \frac{4 \pi^{2} \alpha_{e m} Z_{F}^{2}}{Q^{2}} \int_{\beta}^{1} \int_{\bar{Q}_{0}^{2}}^{\frac{M^{2}}{4}} \frac{d k^{2}}{k^{4}} \frac{4 \alpha_{\mathrm{S}}^{3}}{3 N_{C} \pi} \ln \frac{M^{2}}{4 k^{2}} \\
\beta(1-\beta)^{2}\left\{-2 \beta \ln \beta+\frac{1}{12}\left[1+6 \beta-9 \beta^{2}-24 \beta^{2}-6 \beta^{4}-4 \beta^{5}\right]\right\}\left(x_{P} G\left(k^{2}, x_{P}\right)\right)^{2}
\end{gathered}
$$

One can calculate the one gluon emission, for the longitudinal polarised photon, using the general formula obtained in Ref. [7]

$$
\left.x_{P} \frac{d \sigma_{L}}{d x_{P} d t}\right|_{t=0}(F i g .2 a)=\sum_{F} \frac{4 \pi^{2} \alpha_{e m} Z_{F}^{2}}{Q^{2}} \beta \int_{\beta}^{1} \frac{d z}{z}
$$




$$
\int_{\bar{Q}_{0}^{2}}^{\frac{M^{2}}{4}} \frac{d k^{2}}{k^{4}} \frac{\alpha_{\mathrm{S}}^{3}}{32 \pi} \ln \frac{Q^{2}}{k^{2}} \Phi_{F}^{F}\left(\frac{\beta}{z}\right) \Phi_{P ; T}^{F}(z)\left(x_{P} G\left(k^{2}, x_{P}\right)\right)^{2}
$$

where $\Phi_{F}^{F}$ denotes the usual DGLAP splitting function, and $\Phi_{P}^{F}$ is the splitting function of the Pomeron into a quark-antiquark pair. For the transverse polarisation of the photon this splitting function has been calculated previously in Refs. [5] [7] and we have recalculated it in the $r_{\perp}$ - representation (see eq. (28))

$$
\Phi_{P ; T}^{F}=\frac{16}{N_{C}} z^{2}(1-z)^{2}
$$

For the longitudinal polarization we obtain the same factorized answer with one important difference, the integration over $k^{2}$ is logarithmic (see eq. (23)). However, as far as the $\mathrm{z}$ dependence is concerned, the formula is the same as eq. (38) with the splitting function which we have calculated previously (see eq. (21)) and which is equal to

$$
\Phi_{P ; L}^{F}=\frac{16}{N_{C}} z^{3}(1-2 z)^{2}
$$

Finally, the answer for the one gluon emission in the case of a longitudinal polarised photon is

$$
\begin{gathered}
\left.x_{P} \frac{d \sigma_{L}}{d x_{P} d t}\right|_{t=0}(\text { Fig. } 2 a)=\sum_{F} \frac{4 \pi^{2} \alpha_{e m} Z_{F}^{2}}{Q^{4}} \int_{\beta}^{1} \frac{d z}{z^{6}} \int_{\bar{Q}_{0}^{2}}^{\frac{M^{2}}{4}} \frac{d k^{2}}{k^{2}} \frac{4 \alpha_{\mathrm{S}}^{3}}{3 N_{C} \pi} \ln \frac{Q^{2}}{k^{2}} \\
\beta \frac{1+z^{2}}{1-z}\left\{\beta^{3}(z-2 \beta)^{2}-z^{7} \beta^{3}(1-2 \beta)^{2}\right\}\left(x_{P} G\left(k^{2}, x_{P}\right)\right)^{2}= \\
\sum_{F} \frac{4 \pi^{2} \alpha_{e m} Z_{F}^{2}}{Q^{4}} \int_{\bar{Q}_{0}^{2}}^{\frac{M^{2}}{4}} \frac{d k^{2}}{k^{2}} \frac{4 \alpha_{\mathrm{S}}^{3}}{3 N_{C} \pi} \ln \frac{Q^{2}}{k^{2}} \\
\left\{-2 \beta^{3} \ln \beta(1-2 \beta)^{2}+\frac{2}{15}+\frac{1}{6} \beta+\frac{2}{3} \beta^{2}-4 \beta^{3}+9 \beta^{4}+\frac{74}{15} \beta^{5}-\frac{19}{3} \beta^{6}-\frac{2}{3} \beta^{7}\right\}\left(x_{P} G\left(k^{2}, x_{P}\right)\right)^{2}
\end{gathered}
$$

\section{Shadowing corrections for the diffractive produc- tion of small masses}

The advantage of using the $r_{\perp}$ representation is not apparent in the calculations performed in the previous section, in that we obtain the same results previously obtained by using momentum space calculation techniques (see for example Refs. [5] [6] [7] [20] 24]). The above calculations are instructive and they provide an explicit example of the relations between 
different variables, used to describe deep inelastic process in different frames. In this section, where we calculate the shadowing corrections for the various processes, the $r_{\perp^{-}}$ representation simplifies the calculation. We will only calculate the damping factors for the penetration of a $\bar{q} q$ - pair through the target, assuming that all SC to the gluon density have been included in the phenomenological gluon density that we use in the calculations. The size of the corrections due to damping in the gluon sector has been estimated in Ref. 25].

\subsection{The $b_{\perp}$ dependance of the amplitude}

To estimate the shadowing corrections, we need to know the profile of the amplitude in impact parameter space, which means that we need to know the amplitude not only at $q_{\perp}=0$, but also at all values of the momentum transfer. The gluon density is weakly dependent on $q_{\perp}$ at small values of $q_{\perp}$, both in the double log approximation (see a detailed discussion in Ref. 20]) and in the BFKL approach (see Refs. 27] 28]). Therefore, the leading $q_{\perp}$-dependence comes from the form factor of the $\bar{q} q$ pair with a transverse separation of $r_{\perp}$, and the form factor of the target (proton).

As the form factor cannot be treated theoretically in pQCD, we will assume an exponential parametrization for it, namely

$$
F_{p}\left(q_{\perp}^{2}\right)=e^{-\frac{B}{4} q_{\perp}^{2}}
$$

The slope $B$ can be extracted from the experimental data on hadron-hadron collisions, provided we take the Pomeron slope $\alpha^{\prime}=0$. Namely, $B=B_{e l}^{p p}\left(\alpha^{\prime}=0\right)$, where $B_{e l}$ is the slope in the differential cross section of the proton-proton collision 29]. It turns out that the value of $B$ deduced from the hadronic data is very close to the one obtained from the proton electromagnetic form factor.

The form factor of the $\bar{q} q$ pair with a transverse separation of $r_{\perp}$ is equal to

$$
F_{\bar{q} q}\left(q_{\perp}^{2}\right)=\Psi_{\bar{q} q}^{i}\left(\frac{\left(\vec{k}_{1 \perp}-\vec{k}_{2 \perp}\right) \cdot \vec{r}_{\perp}}{2}\right) \Psi_{\bar{q} q}^{f *}\left(\frac{\left(\vec{k}_{1 \perp}^{\prime}-{\overrightarrow{k^{\prime}}}_{2 \perp}\right) \cdot \vec{r}_{\perp}}{2}\right)
$$

where $k_{i}\left(k_{i}^{\prime}\right)$ denote the momentum of the quark $i$ before and after the collision. Each of the wave functions is given by the exponent and a simple sum of different attachments of gluon lines to quark lines. Hence,

$$
F_{\bar{q} q}\left(q_{\perp}^{2}\right)=e^{i \frac{\vec{q}_{\perp} \cdot \vec{r}_{\perp}}{2}}\left(1-e^{i \vec{l}_{\perp} \cdot \vec{r}_{\perp}}\right)
$$


The last factor is absorbed in the expression for the cross section, while the first factor gives the $q_{\perp}$ dependence of the $\bar{q} q$ form factor, which after integration over the azimuthal angle is

$$
F_{\bar{q} q}\left(q_{\perp}^{2}\right)=J_{0}\left(\frac{q_{\perp} r_{\perp}}{2}\right)
$$

To proceed with the calculation we require the profile function in $b_{\perp}$ space

$$
S\left(b_{\perp}^{2}\right)=\frac{1}{4 \pi^{2}} \int d^{2} q_{\perp} e^{i \vec{b}_{\perp} \cdot \vec{q}_{\perp}} F_{p}\left(q_{\perp}^{2}\right) F_{\bar{q} q}\left(q_{\perp}^{2}\right)
$$

The explicit calculation gives

$$
S\left(b_{\perp}^{2}\right)=\frac{1}{\pi B} I_{0}\left(\frac{b_{\perp} r_{\perp}}{B}\right) e^{-\frac{b_{\perp}^{2}+\frac{r_{\perp}^{2}}{4}}{B}}
$$

To simplify the calculation we replace the above function by

$$
S\left(b_{\perp}^{2}\right)=\frac{1}{\pi B^{\prime}} e^{-\frac{b^{2}}{B^{\prime}}}
$$

where

$$
B^{\prime}=B\left(1+\frac{r_{\perp}^{2}}{4 B}\right) \approx B\left(1+\frac{1}{a^{2} B}\right)
$$

For sufficiently large values of $a$, we consider $a^{2} B \gg 1$, and neglect the second term in our calculation.

\subsection{Penetration of a $\bar{q} q$-pair through the target}

To calculate the shadowing correction we follow the procedure suggested in Refs. [8][9]. Namely, we replace $\sigma\left(r_{\perp}, q_{\perp}^{2}=0\right)$ in eq. (12) by

$$
\sigma^{S C}\left(r_{\perp}\right)=2 \int d^{2} b_{\perp}\left(1-e^{-\frac{1}{2} \sigma\left(r_{\perp}, q_{\perp}^{2}=0\right) S\left(b_{\perp}^{2}\right)}\right)
$$

The above formula is the solution of the s-channel unitarity constraint

$$
2 \operatorname{Im} a\left(s, b_{\perp}\right)=\left|a\left(s, b_{\perp}\right)\right|^{2}+G_{i n}\left(s, b_{\perp}\right)
$$

where $a$ denotes the elastic amlitude for $\bar{q} q$ pair with a transverse separation $r_{\perp}$, while $G_{i n}$ is the contribution of the inelastic processes. The inelastic cross section is equal to

$$
\sigma_{i n}=\int d^{2} b_{\perp} G_{i n}\left(s, b_{\perp}\right)=\int d^{2} b_{\perp}\left(1-e^{-\sigma\left(r_{\perp}, q_{\perp}^{2}=0\right) S\left(b_{\perp}^{2}\right)}\right)
$$


Our formulation is based on the physical assumption that the structure of the final state is obtained from the uniform parton distribution derived from the QCD evolution equation. We neglect the contribution to the inelastic final state of all DD processes with large rapidity gap. For example, "fan" diagrams that give an important contribution are neglected 20] as well as DD in the region of small mass, which cannot be presented as the decomposition of the $\bar{q} q$ wave function.

¿From a point of view of Feynman diagrams, eq. (52) sums all the diagrams of Figs. 1 and 2 in which the $\bar{q} q$-pair rescatters off the target and exchanges many "ladder" diagrams, each of which can be represented by the gluon density. This sum has been performed by Mueller [9], and we shall perform the calculation for the case of small mass DD.

\subsection{Damping factor for diffractive production of small masses induced by a longitudinal photon.}

Substituting $\sigma^{S C}$ of eq. (50) in eq. (12) and using $\sigma$ in the form of eq. (19), enables us to estimate the general term of the expansion with respect to $\sigma$. It has the form

$$
\begin{gathered}
f^{n}=C \frac{k_{\perp}^{2}}{2 Q} \int \frac{d^{2} r_{\perp}}{\pi} K_{0}\left(a r_{\perp}\right) \frac{(-1)^{n-1}}{n !}\left(\frac{\alpha_{\mathrm{S}} 4 C_{F} \pi^{2}}{N_{C}^{2}-1}\right)^{n} \\
\prod_{i}^{n} \int_{C_{i}} \frac{d \omega_{i} e^{\sum \omega_{i} \ln \left(1 / x_{P}\right)}}{2 \pi i} g_{i}\left(\omega_{i}\right) \frac{\Gamma\left(1+\gamma\left(\omega_{i}\right)\right) \Gamma\left(1-\gamma\left(\omega_{i}\right)\right)}{\left(\Gamma\left(2-\gamma\left(\omega_{i}\right)\right)\right)^{2}} \\
\left(\frac{r_{\perp}^{2}}{4}\right)^{n-\sum_{i}^{n} \gamma\left(\omega_{i}\right)} J_{0}\left(k_{\perp} r_{\perp}\right) \int d^{2} b_{\perp} S^{n}\left(b_{\perp}^{2}\right)
\end{gathered}
$$

We replaced all numerical coefficients by the factor $C$.

Integrating over $r_{\perp}$ and $b_{\perp}$ we obtain the expression

$$
\begin{gathered}
f^{n}=C \frac{1}{2 Q} \frac{(-1)^{n-1}}{n n !}\left(\frac{\alpha_{\mathrm{S}} 4 C_{F} \pi}{B^{\prime}\left(N_{C}^{2}-1\right)}\right)^{n} \\
\prod_{i}^{n} \int_{C_{i}} \frac{d \omega_{i} e^{\sum \omega_{i} l n\left(1 / x_{P}\right)}}{2 \pi i} g_{i}\left(\omega_{i}\right) \frac{\Gamma\left(1+\gamma\left(\omega_{i}\right)\right) \Gamma\left(1-\gamma\left(\omega_{i}\right)\right)}{\left(\Gamma\left(2-\gamma\left(\omega_{i}\right)\right)\right)^{2}} \\
\left(\frac{a^{2}}{\beta}\right)^{\sum \gamma\left(\omega_{i}\right)} \Gamma^{2}\left(1+n-\sum_{i}^{n} \gamma\left(\omega_{i}\right)\right)\left(\frac{\beta^{2}}{a^{2}}\right)^{n}{ }_{1} F_{1}\left(1+n-\sum \gamma\left(\omega_{i}\right),-n+\sum \gamma\left(\omega_{i}\right), 1,1-\beta\right)
\end{gathered}
$$


In the double log approximation of pQCD with $\gamma\left(\omega_{i}\right) \ll 1$, neglecting $\gamma$ and taking $1-\beta \ll 1$, we derive a very simple formula. Taking the integral over $\omega_{i}$ yields

$$
f^{n}=C B^{\prime} \frac{\beta^{2}}{2 Q} \frac{(-1)^{n-1} n !}{n}\left(\frac{C_{F} \pi}{B^{\prime}\left(N_{C}^{2}-1\right)} \frac{4 \beta}{a^{2}} \alpha_{\mathrm{S}} x_{P} G\left(\frac{a^{2}}{\beta}, x_{P}\right)\right)^{n}
$$

Finally, for $f$ we have

$$
f=C B^{\prime} \sum_{n=1}^{\infty} \frac{\beta^{2}}{2 Q}(n-1) !\left(\frac{C_{F} \pi}{B^{\prime}\left(N_{C}^{2}-1\right)} \frac{4 \beta}{a^{2}} \alpha_{\mathrm{S}} x_{P} G\left(\frac{a^{2}}{\beta}, x_{P}\right)\right)^{n}
$$

The above series can be summed to give the analytic function $E_{1}$, namely

$$
f=C B^{\prime} \frac{\beta^{2}}{2 Q} E_{1}\left(\frac{1}{\kappa_{q}}\right) e^{\frac{1}{\kappa_{q}}}
$$

where (for $N_{C}=3$ )

$$
\kappa_{q}=\frac{2}{3} \frac{\alpha_{\mathrm{S}} \pi \beta}{B^{\prime} a^{2}} \quad x_{P} G\left(\frac{a^{2}}{\beta}, x_{P}\right)=\frac{2}{3} \frac{\alpha_{\mathrm{S}} \pi(1-\beta)}{B^{\prime} k_{\perp}^{2}} \quad x_{P} G\left(\frac{k_{\perp}^{2}}{(1-\beta)}, x_{P}\right)
$$

Using the above equation, we obtain the following expression for the damping factor

$$
D_{L}^{2}=\frac{\int_{Q_{0}^{2}}^{\frac{M^{2}}{4}} k_{\perp}^{2} d k_{\perp}^{2} E_{1}^{2}\left(\frac{1}{\kappa_{q}}\right) e^{\frac{2}{\kappa_{q}}}}{\int_{Q_{0}^{\frac{M^{2}}{4}}}^{k^{2}} k_{\perp}^{2} d k_{\perp}^{2} \kappa_{q}^{2}}
$$

The behaviour of the damping factor for small and large $\kappa_{q}$ can be found by using the well known property of $E_{1}$. Namely, for $\kappa_{q} \ll 1, D \rightarrow 1$, while at $\kappa_{q} \gg 1$ the damping factor vanishes as

$$
D_{L}^{2} \propto \frac{\int_{Q_{0}^{2}}^{\frac{M^{2}}{4}} k_{\perp}^{2} d k_{\perp}^{2} \ln ^{2} \kappa_{q}}{\int_{Q_{0}^{2}}^{\frac{M^{2}}{4}} k_{\perp}^{2} d k_{\perp}^{2} \kappa_{q}^{2}}
$$

The behaviour of $D_{L}^{2}$ as a function of $\beta$ and $Q^{2}$ is given in Fig.3. One can see that the value of the damping factor reaches 0.5 in the region of small $\beta$ and $Q^{2}$. This means that the SC should be included even for a longitudinal photon, where the smallest distances of the order of $\frac{1}{Q}$ contributes. 


\subsection{Damping factor for diffractive production of small masses induced by a transverse photon}

Repeating the steps of section 3.3 we derive the formula for the damping factor for a transverse polarized photon. Substituting $\sigma^{S C}$ of eq. (50) in eq. (12) we obtain

$$
\begin{gathered}
f^{n}=C a \int \frac{d^{2} r_{\perp}}{\pi} K_{1}\left(a r_{\perp}\right) \frac{(-1)^{n-1}}{n !}\left(\frac{\alpha_{\mathrm{S}} 4 C_{F} \pi^{2}}{N_{C}^{2}-1}\right)^{n} \\
\prod_{i}^{n} \int_{C_{i}} \frac{d \omega_{i} e^{\sum \omega_{i} \ln \left(1 / x_{P}\right)}}{2 \pi i} g_{i}\left(\omega_{i}\right) \frac{\Gamma\left(1+\gamma\left(\omega_{i}\right)\right) \Gamma\left(1-\gamma\left(\omega_{i}\right)\right)}{\left(\Gamma\left(2-\gamma\left(\omega_{i}\right)\right)\right)^{2}} \\
\left(\frac{r_{\perp}^{2}}{4}\right)^{n-\sum_{i}^{n} \gamma\left(\omega_{i}\right)} J_{1}\left(k_{\perp} r_{\perp}\right) \int d^{2} b_{\perp} S^{n}\left(b_{\perp}^{2}\right)
\end{gathered}
$$

$C$ stands for all numerical coefficients.

After integration over $r_{\perp}$ and $b_{\perp}$ we have

$$
\begin{gathered}
f^{n}=C \beta \frac{k_{\perp}}{a^{2}} \frac{(-1)^{n-1}}{n n !}\left(\frac{\alpha_{\mathrm{S}} 4 C_{F} \pi}{B^{\prime}\left(N_{C}^{2}-1\right)}\right)^{n} \\
\prod_{i}^{n} \int_{C_{i}} \frac{d \omega_{i} e^{\sum \omega_{i} l n\left(1 / x_{P}\right)}}{2 \pi} g_{i}\left(\omega_{i}\right) \frac{\Gamma\left(1+\gamma\left(\omega_{i}\right)\right) \Gamma\left(1-\gamma\left(\omega_{i}\right)\right)}{\left(\Gamma\left(2-\gamma\left(\omega_{i}\right)\right)\right)^{2}}\left(\frac{a^{2}}{\beta^{2}}\right)^{\sum \gamma\left(\omega_{i}\right)}\left(\frac{\beta^{2}}{a}\right)^{n} \\
\Gamma\left(2+n-\sum_{i}^{n} \gamma\left(\omega_{i}\right)\right) \Gamma\left(1+n-\sum_{i}^{n} \gamma\left(\omega_{i}\right)\right)_{1} F_{1}\left(2+n-\sum \gamma\left(\omega_{i}\right), 1-n+\sum \gamma\left(\omega_{i}\right), 2,1-\beta\right)
\end{gathered}
$$

Again, in the double log approximation of pQCD with $\gamma\left(\omega_{i}\right) \ll 1$, neglecting $\gamma$ as well as considering $1-\beta \ll 1$, we take the integral over $\omega_{i}$ and get

$$
f^{n}=C B^{\prime} \frac{\beta k_{\perp}}{a^{2}} \frac{(-1)^{n-1} n !(n+1)}{n}\left(\frac{C_{F} \pi}{B^{\prime}\left(N_{C}^{2}-1\right)} \frac{4 \beta}{a^{2}} \alpha_{\mathrm{S}} x_{P} G\left(\frac{a^{2}}{\beta}, x_{P}\right)\right)^{n}
$$

Finally we obtain

$$
f=C B^{\prime} \sum_{n=1}^{\infty} \frac{\beta k_{\perp}}{a^{2}}((n-1) !+n !)\left(\frac{C_{F} \pi}{B^{\prime}\left(N_{C}^{2}-1\right)} \frac{4 \beta^{2}}{a^{2}} \alpha_{\mathrm{S}} x_{P} G\left(\frac{a^{2}}{\beta^{2}}, x_{P}\right)\right)^{n}
$$

The above series can written in terms of the analytic function $E_{1}$

$$
f=C B^{\prime} \frac{\beta k_{\perp}}{2 a^{2}}\left(1+\left(1-\frac{1}{\kappa_{q}}\right) E_{1}\left(\frac{1}{\kappa_{q}}\right) e^{\frac{1}{\kappa_{q}}}\right)
$$


where $\kappa_{q}$ is given by eq. (58).

Using the above equation we get

$$
D_{T}^{2}=\frac{\int_{Q_{0}^{2}}^{\frac{M^{2}}{4}} d k_{\perp}^{2}\left\{1+\left(1-\frac{1}{\kappa_{q}}\right) E_{1}\left(\frac{1}{\kappa_{q}}\right) e^{\frac{1}{\kappa_{q}}}\right\}^{2}}{4 \int_{Q_{0}^{2}}^{\frac{M^{2}}{4}} d k_{\perp}^{2} \kappa_{q}^{2}}
$$

Fig. 4 shows the dependence of $D_{T}^{2}$ on $\beta$ and $Q^{2}$. It is interesting to note that the value of $D_{T}^{2}$ is small for small $\beta$. This reflects a well known fact that the SC are large for DD producing a system with a large mass [10] [20].

\subsection{The relationship between $\mathrm{SC}, F_{2}\left(Q^{2}, x_{B}\right)$ and the DD pro- cesses}

The simplest relation between the corrections to $F_{2}$ and the DD cross section can be derived directly from the AGK cutting rules [26] (see ref.[7]) and it reads

$$
\frac{\Delta F_{2}\left(Q^{2}, x_{B}\right)}{F_{2}\left(Q^{2}, x_{B}\right)}=\frac{\sigma^{D D}}{\sigma_{t o t}}
$$

where

$$
F_{2}=F_{2}^{D G L A P}-\Delta F_{2}
$$

However, eq. (67) is only valid when the DD cross section is small. The notion of what is meant by "small" in diffractive production is not unique, since DD is small in two cases: i) when the kinematic region between partons is very small, this can be dealt with in a perturbative way, or ii) in the case when the interaction is so strong that we have scattering off a black disc. As we have shown, the damping factors for DD turn out to be rather large. It is therefore necessary to reconsider the simple relation in eq. (67).

We generalise eq. (67) by calculating the SC for $F_{2}$ due to the penetration of a $\bar{q} q$ pair through the nucleon. As we have mentioned above, we adopt throughout the paper the approach that the $\mathrm{SC}$ in the gluon sector have been already taken into account in the phenomenological set of densities that we use 15]. The expression for the $F_{2}$ including SC for $\bar{q} q$ pair has been derived by Mueller [9] and in our notation has the following form

$$
F_{2}\left(Q^{2}, x\right)=\sum_{F} Z_{F}^{2} \int_{0}^{1} d z \int \frac{d^{2} r_{\perp}}{2 \pi} \Psi_{T}^{\gamma^{*}}\left(Q^{2}, r_{\perp}, z\right) \sigma^{S C}\left(r_{\perp}\right)\left[\Psi_{T}^{\gamma^{*}}\left(Q^{2}, r_{\perp}, z\right)\right]^{*}
$$


As was shown in ref. 9], whithin the LLA of pQCD which we use throughout this paper, we can safely replace $\Psi_{T} \Psi_{T}^{*}$ by $\frac{1}{r_{\perp}^{4}}$ after integrating over $z$ in eq. (69). Mueller's formula finally reads (for $N_{C}=N_{F}=3$ )

$$
F_{2}\left(Q^{2}, x\right)=\sum_{F} Z_{F}^{2} \frac{6}{\pi^{2}} \int \frac{d^{2} b_{\perp}}{\pi} \int_{\frac{4}{Q^{2}}}^{\infty} \frac{d^{2} r_{\perp}}{\pi} \frac{1}{r_{\perp}^{4}}\left(1-\exp \left(-\frac{1}{2} \sigma\left(r_{\perp}, q_{\perp}^{2}=0\right) S\left(b_{\perp}^{2}\right)\right)\right)
$$

Adopting the same procedure of integration as for eq. (59) and eq. (66), we derive the formula for the elastic damping factor which we define by

$$
D_{e l}^{2}=\frac{\left|\Delta F_{2}\right|}{\left|F_{2}^{N B A}\right|}
$$

where $F_{2}^{N B A}$ denotes the correction to $F_{2}$ due to two Pomeron exchange. In other words, $F_{2}^{N B A}$ is the next order to the Born approximation of eq. (70). The formula for $D_{\text {el }}^{2}$ is

$$
D_{e l}^{2}=\frac{\left|\left(1+\kappa_{q}\right)\left(E_{1}\left(\kappa_{q}\right)+\ln \kappa_{q}+C\right)+1-e^{-\kappa_{q}}-2 \kappa_{q}\right|}{\frac{\kappa_{q}^{2}}{4}}
$$

where $\kappa_{q}$ is defined by eq. (58) at $\frac{a^{2}}{\beta}=Q^{2} . D_{\text {el }}^{2}$ is plotted in Fig.5 for different values of $Q^{2}$. It is interesting to note that $D_{e l}^{2}$ turns out to be very close to 1 over a wide range of $x_{P}$ because $D_{e l}^{2}=1-\frac{\kappa_{q}}{9}$ at small values of $\kappa_{q}$.

Using $D_{e l}^{2}$ and $D_{T}^{2}\left(D_{L}^{2}\right)$ we can rewrite eq. (67) in a more general form

$$
\begin{gathered}
\frac{\Delta F_{2}\left(Q^{2}, x\right)}{F_{2}\left(Q^{2}, x\right)}= \\
\frac{1}{\sigma_{t o t}\left(Q^{2}, x\right)} \int_{0}^{1} d \beta \frac{d \sigma_{T}^{D D}\left(Q^{2}, x, \beta\right)}{d x_{P}} \frac{D_{e l}^{2}}{D_{T}^{2}}+\frac{1}{\sigma_{t o t}\left(Q^{2}, x\right)} \int_{0}^{1} d \beta \frac{d \sigma_{L}^{D D}\left(Q^{2}, x, \beta\right)}{d x_{P}} \frac{D_{e l}^{2}}{D_{L}^{2}}
\end{gathered}
$$

¿From Figs.3 and 4 one can see that the ratio $\frac{D_{e l}^{2}}{D_{T}^{2}}$ can be rather large, and reaches a value of about 2 at low $x$ in the HERA kinematic region.

\section{Numerics and results}

\subsection{What distances are essential in DD processes?}

The first question that we wish to address is, "what are the distances which are important in DD processes?" 
1. In the case of a longitudinal polarised photon, the answer is obvious, just by examining eq. (23). Indeed, in all calculations that lead to eq. (22) and eq. (23) the typical distances are $r_{\perp} \propto \frac{1}{a}=\frac{M}{Q k_{\perp}}$. Since the integral over $k_{\perp}$ in eq. (23) is logarithmic, we conclude that $k_{\perp} \approx M / 2$, from which we estimate that the dominant distances are $r_{\perp} \approx \frac{2}{Q}$. Hence, we can safely utilize the pQCD approach for inclusive production initiated by a longitudinal polarised photon. In Fig. 6 we plot our predictions for $\beta=0.8$. In spite of the $1 / Q^{2}$ suppression we obtain a large value for the cross section. This suggests an alternate way to extract the gluon density, similar to that suggested for vector meson production [4] [6] 25]. It has several advantages: (i) The cross section is larger than that for vector meson production; (ii) The prediction is independent of the form chosen for the nonperturbative wave function of the produced vector mesons, which is an inherent difficultly when making a definite prediction for the DD cross section for vector meson production [25] [30; (iii) The seperation of the longitudinal from the tranverse polarised photon is not difficult. We have not found any contamination fron a transverse polarised photon induced reaction, for events with large values of $\beta$ (practically for $\beta>0.7$ ).

2 . In the case of a transverse polarised photon, the value of the distances for which the pQCD calculation of the DD cross section is valid, depends crucially on the behaviour of the gluon density function at relatively small values of the photon virtuality. Indeed, the cross section of eq. (28) has an extra $k^{2}$ in the denominator, and the integral on first sight appears to be infrared divergent. This is not so, as the gluon density is proportional to $k^{2} / \mu^{2}$ at small values of $k^{2}$. This fact is a direct consequence of the gauge invariance of QCD. However, there is a danger that at very small values of $k^{2}$ in the nonperturbative QCD region, we could still have divergencies as $\mu^{2}$ is about the size of the confiniment radius. We know that $\mu$ depends on $x$, and tends to be large at very small $x$ [9] [20]. The question arises as to what is the situation in the kinematic region of HERA? In an attempt to answer this question we adopt the following strategy: We believe that the available parametrizations of the DIS structure functions (GRV [15], MRS[31], CTEQ [33]) describe the behaviour of the gluon density in the region of relatively small virtualities. The common feature of all these parametrization is the fact that the behaviour $x G\left(k^{2}, x\right) \propto k^{2}$ starts at virtualities which are sufficiently large in the small $x$ region. For larger $x$ and at larger values of $k^{2}, x G\left(k^{2}, x\right)$ starts to be proportional to $k\left(x G\left(x, k^{2}\right) \propto k / \mu\right)$. For example, at $Q^{2}=2.5 \mathrm{GeV}^{2}$, in the GRV parametrization, $x G\left(x, k^{2}\right) \propto k / \mu$ at $x<3.10^{-3}$, while in the $\operatorname{MRS}(\mathrm{A})$ this happens at $x<10^{-2}$. Relying on the available sets of parton densities (which contain all accumulated experimental information on the subject), we conclude that even for a transverse polarized photon, the typical distances in the DD processes are not larger than $r_{\perp} \approx 1 G e V^{-1}$. Hence, we can apply the pQCD approach in this case as well. 
To illustrate this we plot in Fig.7 the integrand of eq. (28)

$$
I\left(k^{2}, x_{P}, \beta\right)=\frac{\left(x_{P} G\left(\frac{k_{\perp}^{2}}{1-\beta}, x_{P}\right)\right)^{2}}{k_{\perp}^{2}}\left(1-2 \frac{k_{\perp}^{2}}{M^{2}}\right) \frac{1}{\sqrt{1-\frac{4 k_{\perp}^{2}}{M^{2}}}}
$$

as a function of $x_{P}$ and $k^{2}$ at fixed $\beta$. We wish to emphasise that the integrand can be viewed as the product of two factors: $\left(x_{P} G\left(\frac{k_{\perp}^{2}}{1-\beta}, x_{P}\right)\right)^{2} / k_{\perp}^{2}$ and the kinematic factor $\left(1-2 \frac{k_{\perp}^{2}}{M^{2}}\right) \frac{1}{\sqrt{1-\frac{4 k_{\perp}^{2}}{M^{2}}}}$. The first factor has a maximum at $k_{\perp}^{2} \approx 1 \mathrm{GeV}^{2}$ for all available parametrizations of the gluon density. The origin of this maximum is very simple and can easily be traced if one uses the semiclasical parametrisation of the solution to the evolution equations in the form

$$
x_{P} G\left(k^{2}, x_{P}\right) \rightarrow\left(\frac{1}{x_{P}}\right)^{\omega\left(k^{2}, x_{P}\right)}\left(k^{2}\right)^{\gamma\left(k^{2}, x_{P}\right)} \text { at } x_{P} \rightarrow 0
$$

where both $\omega$ and $\gamma$ are smooth functions of $\ln \left(1 / x_{P}\right)$ and $\ln k^{2}$. The common feature of the various parametrisations of the gluon density is the fact that $\gamma>\frac{1}{2}$ at $x_{P} \leq 10^{-3}$. Such a behaviour manifests itself in a maximum of the first factor at $k^{2} \approx 1 \mathrm{GeV}^{2}$. It should be stressed that the argument of the gluon density in eq. (28), namely $k_{\perp}^{2} /(1-\beta)$, leads to a substantial increase of the typical transverse momentum in the integral. This amounts to a decrease of the typical value of the distances important for diffractive $\bar{q} q$ production. The kinematic factor also tends to increase at large values of $k^{2}$.

Finally, we would like to repeat our statement that the DD process initiated by either longitudinal or transverse polarised photons occur at small distances. This fact is essential to justify the use of pQCD for the calculation of DD.

\subsection{The $x_{P}$ dependence, factorization and the "Pomeron struc- ture function"}

The $x_{P}$ dependence of the calculated $F_{2}^{D D(3)}$ is plotted in Fig.8 together with the H1 data [14], where

$$
x_{P} F_{2}^{D D(3)}\left(Q^{2}, x_{P}, \beta\right)=\frac{Q^{2}}{4 \pi^{2} \alpha_{e m}} \int d t x_{P} \frac{d \sigma}{d x_{P} d t}=\left.\frac{Q^{2}}{4 B \pi^{2} \alpha_{e m}} x_{P} \frac{d^{2} \sigma}{d x_{P} d t}\right|_{t=0}
$$

and $B$ denotes the slope of the DD cross section. We take $B=4.5 \mathrm{GeV}^{-2}$ in accord with the the prelimenary experimental data from HERA (see Ref. 35]). The value of $B$ has 
not yet been measured with good accuracy. The above value of $B$ coincide with the slope that has been extracted from the high energy phenomenology of "soft" processes (see, for example, Ref.[29]).

We wish to stress that in all our comparisons with the experimental data, including Fig.8, we include the SC, which have been calculated in the previous section. This means that the formulae for the cross sections, obtained in section 2, are multiplied by the damping factor $D_{L}^{2}$ or $D_{T}^{2}$, calculated in section 3 .

We now concentrate on the $x_{P}$ dependence of $F_{2}^{D D(3)}$. Based on the Ingelman-Schlein hypothesis of the Pomeron structure function[34], $F_{2}^{D D(3)}$ can be written in a factorised form

$$
F_{2}^{D D(3)}=f\left(x_{P}\right) F_{2}^{P}\left(Q^{2}, \beta\right)
$$

where $F_{2}^{P}$ denotes the Pomeron structure function and $f\left(x_{P}\right)$ the Pomeron flux factor. If the Pomeron is a simple Regge pole then $f\left(x_{P}\right) \propto\left(\frac{1}{x_{P}}\right)^{1+n} \cdot n$ is intimately related to the intercept of the Pomeron trajectory: $\alpha_{P}=\alpha_{p}(0)+\alpha_{P}^{\prime}|t|$, where $\alpha_{P}(0)=1+\epsilon$. Thus: $n=2 \alpha_{P}(0)-2=2 \epsilon$.

Our approach is orthogonal to the IS one. We do not expect eq. (77) to scale, and if we use the parametrisation suggested in eq. (77), and fit the $x_{P}$ dependence assuming the form

$$
F_{2}^{D D(3)}=\left(\frac{1}{x_{P}}\right)^{n} F_{2}^{P}\left(Q^{2}, \beta\right)
$$

we expect $n$ to depend on both $\beta$ and $Q^{2}$.

We present the calculated values of $n\left(Q^{2}, \beta\right)$ in Fig.9a. We would like to point out that the value of $n$ is much bigger $(n \approx 0.55)$ than the experimentally fitted value of $(n \approx 0.2)$ (see Ref.[14]). The difference is due to the steep behaviour of the gluon density function in the GRV parametrisation. Notice, however, that the new ZEUS data 13] gives a value of $n \approx 0.4$ which is much closer to our result. Direct comparison with the experimental data given in Fig.9a, shows that our calculation is able to describe the experimental data at $x_{P} \approx 10^{-3}$ but it substantially overshoots the experimental data for smaller values of $x_{P}$. We think this behaviour is an artifact of the GRV parametrisation in the leading order of pQCD. A simple ad hoc procedure to improve our results would be to take GRV in the next to leading order. In our opinion such a procedure is not self consistent unless the entire calculation is done in a higher order. One can see from Fig.9b, where the ratio of the leading order GRV gluon density to the next to leading order distribution $\left(x_{P} G\left(Q^{2}, x_{P}\right)^{L O} / x_{P} G\left(Q^{2}, x_{P}\right)^{N L O}\right)$ is plotted that the use of $x_{P} G\left(Q^{2}, x_{P}\right)^{N L O}$ instead of $x_{P} G\left(Q^{2}, x_{P}\right)^{L O}$ suppresses the value of the diffractive cross section at small $x_{P}$. It is interesting to note that the effective power $n$ reduces then to a value $n \approx 0.3$ 
which is closer to the experimental value suggested by the new ZEUS data 13. The second source of suppression at low $x_{P}$ comes from the energy behaviour of the slope $B$, where $B=B_{0}+2 \alpha_{P}^{\prime} \ln \left(1 / x_{P}\right)$. Note that for a Regge soft Pomeron we have $\alpha_{P}(t)=1+\epsilon+\alpha_{P}^{\prime} t$.

Regardless of the above, the fact that pQCD calculations predict a larger cross section than the experimental one, lends support to our statement in the previous subsection that at small distances, where we can trust the pQCD approach, the calculation of the diffractive production of small mass, appears to work. How the $x_{P}$ dependence is affected by the different parametrizations of the structure function is still an open question. We plan on clarifying this in a future publication.

\subsection{The diffractive structure function $F_{2}^{D D(2)}$}

In Fig.10 we display our calculated values for $F_{2}^{D(2)}$ defined as

$$
F_{2}^{D D(2)}=\int_{\left(x_{P}\right)_{\min }}^{\left(x_{P}\right)_{\max }} d x_{P} F_{2}^{D D(3)}
$$

where the values of $\left(x_{P}\right)_{\min }$ and $\left(x_{P}\right)_{\max }$ are taken to have the same values as in the relevant experiments. In Fig. 11 we compare the calculated $F_{2}^{D D(2)}$ values with the relevant H1 data [14. ¿From these figures we are able to conclude:

1. The pQCD calculation provides a fair description of the experimental data.

2. The contribution of the longitudinal polarised photon is important at large $\beta>0.7$.

3. The process with an emission of an extra gluon gives rise to a sizeble contribution for $\beta<0.4$ and should be taken into account in a more consistent manner by solving the evolution equations for DD processes (see the next section).

4. For $\beta>0.4$, the emission of an extra gluon provides only a small contribution, so one can attempt to extract the gluon density by measuring $F_{2}^{D D(2)}$ at large $\beta$.

\subsection{The transverse momentum spectra}

Our formulae ,see eqs. (23),(28),(36),(37) and (41), can be used for a more detailed analysis of the DD events. In particular, we can describe the transverse momentum spectra of the parton jets in DD. Basically, these spectra are given by the integrand of our formulae, as the $k_{\perp}$ which appears is just the transverse momentum of the produced jet. 
In Fig. 12, the ratio

$$
R=\frac{F_{2}^{D D(2)}\left(k_{\perp}^{2}>k_{0}^{2}\right)}{F_{2}^{D D(2)}}
$$

is presented with different values of $k_{0}^{2}$. The physical meaning of this ratio is that it indicates the fraction of the all DD events possessing transverse momenta larger than $k_{0}$. One can see from Fig.12 that we expect a fairly large fraction of the events with big transverse momenta, this is in agreeement with the new H1 data 36].

In Fig.13 we plot the calculated value

$$
x_{P} F_{2}^{D D(4)}=\left.\frac{Q^{2}}{4 \pi^{2} B \alpha_{e m}} \frac{x_{P} d^{3} \sigma}{d x_{P} d t d k_{\perp}^{2}}\right|_{t=0}
$$

where $k_{\perp}$ denotes the transverse momentum of the jet. The comparison with the experimental data is also quite good, at least, the $k_{\perp}$ distribution reproduces the main qualitative feature of the experimental data [36], namely, smoother behaviour than $\frac{1}{k_{\perp}^{4}}$ at small values of $k_{\perp}^{2}$, and $\frac{1}{k_{\perp}^{4}}$ behaviour for larger $k_{\perp}$. On the other hand, we predict a sharp drop at large $k_{\perp}$, which has not been seen in the data as yet. Perhaps, the emission of two gluons starts to be important at such large values of transverse momenta.

\subsection{Matching with diffractive vector meson electroproduction}

Vector meson diffractive electroproduction corresponds to the kinematic region of $\beta \rightarrow 1$, namely

$$
\beta=\frac{Q^{2}}{Q^{2}+M_{V}^{2}} \rightarrow 1-\frac{M_{V}^{2}}{Q^{2}}
$$

where $M_{V}^{2}$ is the mass of the produced vector meson. In this kinematic region we can rewrite eq. (23) and eq. (28) in the form

$$
\begin{gathered}
\left.M^{2} \frac{d \sigma^{L}}{d M^{2} d t}\right|_{t=0}= \\
\frac{4 \pi^{2} \alpha_{e m} \alpha_{S}^{2}}{3} \sum_{F} Z_{F}^{2} \frac{M_{V}^{2}}{Q^{6}} \int_{Q_{\min }^{2}}^{\frac{M_{V}^{2}}{4}} \frac{d k_{\perp}^{2}}{k_{\perp}^{2}} \frac{1}{\sqrt{1-\frac{4 k_{\perp}^{2}}{M_{V}^{2}}}}\left(x_{P} G\left(\frac{k^{2} Q^{2}}{M_{V}^{2}}, x_{P}\right)\right)^{2} \\
\left.M^{2} \frac{d \sigma^{T}}{d M^{2} d t}\right|_{t=0}=
\end{gathered}
$$




$$
\frac{4 \pi^{2} \alpha_{e m} \alpha_{\mathrm{S}}^{2}}{3} \sum_{F} Z_{F}^{2} \frac{M_{V}^{3}}{Q^{8}} \int_{Q_{m i n}^{2}}^{\frac{M_{V}^{2}}{4}} \frac{d k_{\perp}^{2}}{k_{\perp}^{4}} \frac{1}{\sqrt{1-\frac{4 k_{\perp}^{2}}{M_{V}^{2}}}}\left\{1-2 \frac{k_{\perp}^{2}}{M_{V}^{2}}\right\}\left(x_{P} G\left(\frac{k^{2} Q^{2}}{M_{V}^{2}}, x_{P}\right)\right)^{2}
$$

These formulae give the cross sections for production of all hadrons with mass $M_{V}$, and reproduce the main features of the exclusive vector meson production [3] [4⿴囗十 25 . It is interesting to calculate the cross sections in the region of $M_{V}^{2}=m_{\rho}^{2}$ where we do not expect any other mesons, besides the $\rho$-meson to be produced. In the double log approximation of pQCD, we can put the argument of the gluon density equal to $Q^{2}$. Evaluating the remaining integrals we obtain

$$
\frac{\sigma^{L}}{\sigma^{T}}=\frac{Q^{2} Q_{\min }^{2} \ln \frac{M_{V}^{2}}{4 Q_{\min }^{2}}}{M_{V}^{4}}
$$

The value we take for $Q_{\min }^{2}$ depends on how strong our belief in hadron-parton duality is. If we believe that the hadron-parton duality can be used at large distances, we can choose the low limit of integration from the condition that the argument of the gluon density in eq. (82) and in eq. (83) is bigger than the intitial virtuality in the GRV parametrization, i.e. $Q_{0}^{2}=Q_{\min }^{2} Q^{2} / M_{V}^{2}$ or $Q_{\min }^{2}=Q_{0}^{2} M_{V}^{2} / Q^{2}$. For such a value of $Q_{\min }^{2}$ we have $\sigma^{L} / \sigma^{T}=$ $\frac{Q_{0}^{2}}{M_{V}^{2}} \ln \frac{4 Q^{2}}{Q_{0}^{2}}$.

Experience gained from $e^{+} e-$ annihilation indicates that $Q_{\min }^{2}$ could be very small, about $m_{\pi}^{2}$ [37]. In this case, we still have the $Q^{2}$ rise of the ratio, but the coefficient is very small, $\sigma^{L} / \sigma^{T}=0.125 Q^{2}$. In Fig.14 we plot $\sigma^{L}$ and $\sigma^{T}$, using eq. (82) and eq. (83) with a cutoff $Q_{\min }^{2}=m_{\pi}^{2}$.

Concluding this section, we would like to reiterate our claim that the simple pQCD calculation provides a reliable basis for the discussion of the origin of DD in DIS. It also suggests a new way to determine the gluon density. This new measurement can be done in two unambigous ways:

1. the measurement of $F_{2}^{D D(2)}$ with $\beta>0.7$, allows us to extract the longitudinal polarised photon structure function which is only sensitive to small distances of the order $r_{\perp} \approx$ $1 / Q$.

2. the measurement of $F_{2}^{D D(2)}$ with $k_{\perp}>k_{0} \geq 1 \mathrm{GeV}^{2}$. In this case only distances $r_{\perp} \leq 1 / k_{0}$ contribute to our formulae, and we have reliable pQCD predictions for the fraction of the DD events.

\section{Evolution equations for DD}

In this section we discuss the evolution equations for the DD structure functions that have been introduced in the previous section. The evolution equations for the transverse 
polarised photon have been proposed and derived in Ref.[7], we will comment on them later. We first discuss the evolution equations for the longitudinal polarised photon, which have not been formulated previously. These provide an interesting theoretical insight into the structure of the evolution of exclusive processes in QCD.

\subsection{Evolution equation for DD of a $\bar{q} q$-pair production by a lon- gitudinal photon}

We start with the evolution equation for the simple case of $\bar{q} q$ production (see eq. (23)). It is useful to introduce the DD structure function[]] absorbing all extra powers of $Q^{2}$ in the definition

$$
\begin{gathered}
F_{L}^{D D}=\left.x_{P} \frac{d \sigma}{d x_{P} d t}\right|_{t=0}\left\{4 \pi \alpha_{e m} \sum Z_{F}^{2} \frac{1}{Q^{4}}\right\}^{-1}= \\
\frac{\alpha_{S}^{2}}{N_{C}} \beta^{2}(1-\beta)(1-2 \beta)^{2} \int_{Q_{0}^{2}}^{\frac{M^{2}}{4}} \frac{d k_{\perp}^{2}}{k_{\perp}^{2}}\left(x_{P}, G\left(\frac{k_{\perp}^{2}}{(1-\beta)}, x_{P}\right)\right)^{2}
\end{gathered}
$$

Taking the derivative with respect to $\ln Q^{2}$, we obtain the evolution equation

$$
\frac{\partial F_{L}^{D D}\left(Q^{2}, x_{P}, \beta\right)}{\partial \ln Q^{2}}=\frac{\alpha_{S}^{2}}{N_{C}} \beta^{2}(1-\beta)(1-2 \beta)^{2}\left(x_{P} G\left(\frac{Q^{2}}{4 \beta}, x_{P}\right)\right)^{2}
$$

We first investigate eq. (86) by considering the contribution of the diffractive processes to the deep inelastic structure function (i.e. to the total cross section). We integrate eq. (86) as well as eq. (85) over all values of $1>x_{P}>x_{B}$. The solution of the evolution equation for the integrated $F_{L}^{D D}\left(Q^{2}, \beta\right)\left(F_{L ; i n t}^{D D}=\int_{x_{B}}^{1} d x_{P} F_{L}^{D D}\left(Q^{2}, x_{P}, \beta\right)\right)$ can be obtained by transforming to the moment representation with respect to $\ln \left(1 / x_{B}\right)$ (see eq. (2)). This has the form

$$
\frac{d f_{L ; i n t}^{D D}\left(Q^{2}, \omega\right)}{d \ln Q^{2}}=\alpha_{\mathrm{S}}^{2} f_{L ; i n t}^{G^{2}}\left(Q^{2}, \omega\right)
$$

where we denote by $f^{G^{2}}\left(Q^{2}, \omega\right)$ the moment of the right hand side of eq. (86) integrated over $x_{P}$. Our calculation is done in the double log approximation where we know that 20 38

$$
f_{L ; i n t}^{G^{2}}\left(Q^{2}, \omega\right)=f^{G^{2}}\left(Q_{0}^{2}, \omega\right) e^{\gamma_{2}(\omega) \ln \frac{Q^{2}}{Q_{0}^{2}}}
$$


and

$$
\gamma_{2}(\omega)=\frac{4 N_{C} \alpha_{\mathrm{S}}}{\pi} \frac{1}{\omega} \gg \gamma_{1}(\omega)=\gamma(\omega)=\frac{N_{C} \alpha_{\mathrm{S}}}{\pi} \frac{1}{\omega}
$$

The solution of eq. (87) is

$$
f_{L ; i n t}^{D D}\left(Q^{2}, \omega\right)=\alpha_{S} \frac{f^{G^{2}}\left(Q_{0}^{2}, \omega\right)}{\gamma_{2}(\omega)} e^{\gamma_{2}(\omega) \ln \frac{Q^{2}}{Q_{0}^{2}}}
$$

For the deep inelastic structure function, the process of DD initiated by a longitudinal polarized photon contributes to the twist four term in the Wilson Operetor Product Expansion (note the additional factor of $\frac{1}{Q^{2}}$ in eq. (23)). We claim that the anomalous dimension of the twist four operator is equal to $\gamma_{2}$. It should be stressed that the diffractive cross section does not describe the complete twist four contribution to the structure function. The additional gluon emission which increases the value of the total anomalous dimension 38 does not contribute to the diffraction into small masses.

\subsection{Evolution of the DD structure function (greneral consider- ations)}

In the general case we can rewrite eq. (85) in the form

$$
F_{L}^{D D}=\frac{\alpha_{S}^{2}}{N_{C}} \int_{Q_{0}^{2}}^{Q^{2}(1-\beta)} \frac{d k_{\perp}^{2}}{k_{\perp}^{2}} \int_{\beta}^{1} d z \frac{\beta}{z} \Sigma_{S}^{S}\left(\frac{\beta}{z}, \ln \frac{Q^{2}}{k_{\perp}^{2}}\right) \Phi_{P ; L}^{F}\left(x_{P} G\left(\frac{k_{\perp}^{2}}{(1-\beta)}, x_{P}\right)\right)^{2}
$$

where $\Sigma$ denotes the singlet structure function of the quark-antiquark pair $\left(q\left(x, Q^{2}\right)+\right.$ $\left.\bar{q}\left(x, Q^{2}\right)\right)$ and the Pomeron splitting function $\Phi_{L}^{F}$ has been defined above (see eq. (40)). It should be stressed that only $\Sigma_{S}^{S}$ enters the evolution equation in the leading order of pQCD.

Differentiating the above equation we obtain the evolution equations for the monents in the form

$$
\begin{gathered}
\frac{d f_{L ; i n t}^{D D ; S}\left(Q^{2}, \omega\right)}{d \ln Q^{2}}= \\
P_{S}^{S}(\omega) f_{L ; i n t}^{D D ; S}\left(Q^{2}, \omega\right)+P_{G}^{S}(\omega) f_{L ; \text { int }}^{D D ; G}\left(Q^{2}, \omega\right)+\frac{\alpha_{\mathrm{S}}^{2}}{N_{C}} f^{G^{2}}\left(Q^{2}, \omega\right)
\end{gathered}
$$

where $P$ are the kernels of the DGLAP evolution equations. We obtain the ordinary DGLAP evolution equations with an inhomogenious term. This equation has the solution

$$
f_{L ; i n t}^{D D}\left(Q^{2}, \omega\right)=
$$




$$
\begin{gathered}
\frac{\alpha_{\mathrm{S}}^{2}}{N_{C}} f^{G^{2}}\left(Q_{0}^{2}, \omega\right)\left\{\frac{1}{\gamma_{2}(\omega)-\gamma^{+}(\omega)}+\frac{1}{g_{2}(\omega)-\gamma^{-}(\omega)}\right\} e^{\gamma_{2}(\omega) \ln \frac{Q^{2}}{Q_{0}^{2}}} \\
+f_{L ; i n t}^{D D}\left(Q_{0}^{2}, \omega\right)\left\{\frac{-\gamma^{-}(\omega)}{\gamma^{+}(\omega)-\gamma^{-}(\omega)} e^{\gamma^{+}(\omega) \ln \frac{Q^{2}}{Q_{0}^{2}}}+\frac{\gamma^{+}(\omega)}{\gamma^{+}(\omega)-\gamma^{-}(\omega)} e^{\gamma^{-}(\omega) \ln \frac{Q^{2}}{Q_{0}^{2}}}\right\}
\end{gathered}
$$

where the functions $f_{L ; i n t}^{D D}\left(Q_{0}^{2}, \omega\right)$ and $f^{G^{2}}\left(Q_{0}^{2}, \omega\right)$ denote the intial conditions for the evolution equations, and describes the large distance physics. It is obvious that the second term of eq. (93) is the solution of the DGLAP equations for fixed $\alpha_{\mathrm{S}}$, which we assumed in order to simplify the solution. One can find this form of the solution in Refs. [39] [40] and in the references contained therein. $\gamma^{+,-}$denotes the eigenvalues of the anomalous dimensions matrix and in the leading order of pQCD they are equal to

$$
\gamma^{ \pm}=\frac{1}{2}\left\{P_{S}^{S}+P_{G}^{G} \pm \sqrt{\left(P_{S}^{S}-P_{G}^{G}\right)^{2}+4 P_{G}^{S} P_{S}^{G}}\right\}
$$

where

$$
\begin{gathered}
P_{S}^{S}=\frac{C_{F} \alpha_{S}}{2 \pi}\left\{\frac{3}{2}-\frac{1}{\omega+1}-\frac{1}{\omega+2}-2[\psi(\omega+1)-\psi(1)]\right\} \\
P_{G}^{G}=\frac{\alpha_{S}}{2 \pi}\left\{2 N_{C}\left[\frac{11}{12}+\frac{1}{\omega}-\frac{2}{\omega+1}+\frac{1}{\omega+2}-\frac{1}{\omega+3}-\psi(\omega+1)+\psi(1)\right]-\frac{2 T_{R} N_{F}}{3}\right\} \\
P_{S}^{G}=\frac{C_{F} \alpha_{S}}{2 \pi}\left\{\frac{2}{\omega}-\frac{2}{\omega+1}+\frac{1}{\omega+2}\right\} \\
P_{G}^{S}=\frac{2 T_{R} N_{F} \alpha_{S}}{2 \pi}\left\{\frac{2}{\omega+3}-\frac{2}{\omega+2}+\frac{1}{\omega+1}\right\}
\end{gathered}
$$

It should be stressed that in pQCD only $\gamma^{+}$has a singularity in the second term at small $\omega$, and $\gamma^{+} \ll g_{2}(\omega)$ at $\omega \rightarrow 0$. This means that only the first term survives at small $x$.

We do not claim that this result is valid at small virtualities, where pQCD arguments are not applicable. The value of the DD structure function at a small vituality of the photon $\left(Q^{2}=Q_{0}^{2}\right)$ which can be measured experimentally, defines only the specific sum of singularities, namely

$$
\begin{gathered}
f_{L ; \text { int }}^{D D ; S}\left(Q_{0}^{2}, x_{B}\right)= \\
\frac{1}{2 \pi i} \int_{C} d \omega\left(\frac{\alpha_{\mathrm{S}}^{2}}{N_{C}} f^{G^{2}}\left(Q_{0}^{2}, \omega\right)\left[\frac{1}{\gamma_{2}(\omega)-\gamma^{+}(\omega)}+\frac{1}{\gamma_{2}(\omega)-\gamma^{-}(\omega)}\right]+f_{L}^{D D ; S}\left(Q_{0}^{2}, \omega\right)\right)
\end{gathered}
$$

The fact that these two terms have different anomalous dimensions, suggests that one might be able to use their $x_{P}$ dependence in order to distinguish between them.

The second term is proportional to $\left(x_{P} G\left(Q_{0}^{2}, x_{P}\right)\right)^{2}$ for the unintegrated $F^{D D}$, but there is no theoretical argument why such a behaviour should be valid for a small value 
of $Q_{0}$. Phenomenologically, we associate these two contributions with the "soft" and "hard" Pomeron. This terminology reflects our hope that their $x_{P}$ behaviour is different. Assuming that their behaviour can be approximately parameterized as $x_{P}^{2 \epsilon}$, we expect for the "soft" (second) term $\epsilon_{\text {soft }} \sim 0.08-0.1$, while for the "hard" one (first term in eq. (93)) $\epsilon_{\text {hard }} \sim 0.2-0.5$. We wish to stress that the problem of separating these two contributions is beyond the scope of pQCD, and has to be tackled using a nonperturbative approach. As a guide to phenomenological applications we would like to mention that the Ingelman and Schlein approach 34 based on the Pomeron structure function, means that only the "soft" contribution survives, while the "hard" Pomeron approach deals only with the first tem in eq. (93). Returning to $F^{D D}$ (see eq. (91)), we can write the evolution equation at fixed $x_{P}$

$$
\begin{gathered}
\frac{\partial F_{L}^{D D ; S}\left(Q^{2}, x_{P}, \beta\right)}{\partial \ln Q^{2}}=\int_{\beta}^{1} d z P_{S}^{S}(z) F_{L}^{D D ; S}\left(Q^{2}, x_{P}, \frac{\beta}{z}\right)+ \\
\int_{\beta}^{1} d z P_{G}^{S}(z) F_{L}^{D D ; G}\left(Q^{2}, x_{P}, \frac{\beta}{z}\right)+\frac{\alpha_{S}^{2}}{N_{c}} \beta^{2}(1-2 \beta)^{2}\left(x_{P} G\left(\frac{Q^{2}}{4 \beta}, x_{P}\right)\right)^{2}
\end{gathered}
$$

In moment space with respect to $\ln (1 / \beta)$, the equation reduces to the form

$$
\begin{gathered}
\frac{d f_{L}^{D D ; S}\left(Q^{2}, x_{P}, \omega\right)}{d \ln Q^{2}}= \\
P_{S}^{S}(\omega) f_{L}^{D D ; S}\left(Q^{2}, x_{P}, \omega\right)+P_{G}^{S}(\omega) f_{L}^{D D ; G}\left(Q^{2}, x_{P}, \omega\right)+T(\omega)\left(x_{P} G\left(Q^{2}, x_{P}\right)\right)^{2}
\end{gathered}
$$

where

$$
T_{G ; L}^{F}(\omega)=\frac{1}{\omega+2}-\frac{4}{\omega+3}+\left.\frac{4}{\omega+4} \rightarrow\right|_{\omega \rightarrow 0} \frac{1}{6}
$$

The general solution of the above equation is

$$
\begin{gathered}
f_{L ; i n t}^{D D}\left(Q^{2}, \omega\right)= \\
\frac{\alpha_{\mathrm{S}}^{2}}{N_{C}}\left\{f_{L ; \text { int }}^{D D}\left(Q_{0}^{2}, \omega\right)\left\{\frac{-\gamma^{-}(\omega)}{\gamma^{+}(\omega)-\gamma^{-}(\omega)} e^{\gamma^{+}(\omega) \ln \frac{Q^{2}}{Q_{0}^{2}}}+\frac{\gamma^{+}(\omega)}{\gamma^{+}(\omega)-\gamma^{-}(\omega)} e^{\gamma^{-}(\omega) \ln \frac{Q^{2}}{Q_{0}^{2}}}\right\}\right. \\
+\left[\frac{\gamma_{2}(\omega)}{\gamma_{2}(\omega)-\gamma^{+}(\omega)}+\frac{\gamma_{2}(\omega)}{\gamma_{2}(\omega)-\gamma^{-}(\omega)}\right] \int_{Q_{0}^{2}}^{Q^{2}} \frac{d Q^{\prime 2}}{Q^{\prime 2}} T(\omega)\left(x_{P} G\left(Q^{2}, x_{P}\right)\right)^{2} \\
=f_{S O F T}^{D D}+f_{H A R D}^{D D}
\end{gathered}
$$

The first term $F_{S O F T}^{D D}$ is associated with soft diffraction, and it requires the phenomenological input $f^{D D}\left(\omega, x_{P}, Q_{0}^{2}\right)$. We choose for this input

$$
f^{D D}\left(Q_{0}^{2}, x_{P}, \omega\right)=T(\omega)\left(x_{P} G\left(Q^{\prime 2}, x_{P}\right)\right)^{2}
$$


We still need to fix the value of $Q_{0}$ in our initial condition. There is no reason to assume the same value of $Q_{0}$ in DD and DIS. We choose, for DD, a value of $Q_{0}^{2}$ which is approximately $1-2 \mathrm{GeV}^{2}$. In doing so we can use pQCD to evaluate the two terms appearing in eq. (100), and this leads to eq. (101) for the first term.

In the $\beta$-representation the intial condition is simply

$$
F_{L}^{D D}\left(Q_{0}^{2}, x_{P}, \beta\right)=\frac{\alpha_{\mathrm{S}}^{2}}{N_{C}} \beta^{2}(1-2 \beta)^{2}\left(x_{P} G\left(\frac{Q_{0}^{2}}{4 \beta}, x_{P}\right)\right)^{2}
$$

This is plotted in Fig.15 for different values of $x_{P}$.

\subsection{Evolution equation for transverse polarized photon initiated DD}

The evolution equations for a transverse polarised photon have been given in Ref.[7]. Introducing the densities $q_{P}\left(Q^{2}, x\right)$ and $G_{P}\left(Q^{2}, x\right)$ which denote the quark and gluon densities in the Pomeron, we can rewrite the evolution equations in the form (see ref. [7] for details):

$$
\begin{gathered}
\frac{\partial \Sigma_{P}\left(Q^{2}, x\right)}{\partial \ln Q^{2}}= \\
\int_{\beta}^{1} \frac{d z}{z} P_{S}^{S}(z) \Sigma_{P}\left(Q^{2}, \frac{\beta}{z}\right)+P_{G}^{S}(z) G_{P}\left(Q^{2}, \frac{\beta}{z}\right)+\frac{1}{Q^{2}} \Phi_{P ; T}^{q}(\beta)\left(x_{P} G\left(Q_{0}^{2}, x_{P}\right)\right)^{2} \\
\frac{\partial G_{P}\left(Q^{2}, x\right)}{\partial \ln Q^{2}}=\int_{\beta}^{1} \frac{d z}{z} \\
\left\{P_{S}^{G}(z) \Sigma_{P}\left((1-\beta) Q^{2}, \frac{\beta}{z}\right)+P_{G}^{G}(z) G_{P}\left((1-\beta) Q^{2}, \frac{\beta}{z}\right)\right\} \\
+\frac{1}{Q^{2}} \Phi_{P ; T}^{G}(\beta)\left(x_{P} G\left(Q_{0}^{2}, x_{P}\right)\right)^{2}
\end{gathered}
$$

where $\Sigma=q\left(Q^{2}, x\right)+\bar{q}\left(Q^{2}, x\right)$.

On inspection we note that the structure of the equations is the same as for the longitudinal polarised photon, eq. (103), and is a normal DGLAP evolution equation

with an inhomogenious term. $P$ are the kernels of the DGLAP equations while $\Phi$ denotes the Pomeron splitting functions

$$
\Phi_{P ; T}^{S}=\frac{\alpha_{\mathrm{S}}}{N_{C}} z^{2}(1-z)^{2}
$$




$$
\Phi_{P ; T}^{G}=\frac{\alpha_{\mathrm{S}} N_{C}^{2}}{2\left(N_{C}^{2}-1\right)} \frac{1}{z}(1-z)^{2}(1+2 z)^{2}
$$

The set of eq. (103) can be solved in the moment representation using the standard techniques (see Refs. 39] [40] and references therein). The main difference in the comparison with the lomgitudinal photon, is the fact that the inhomogenious term has an extra $1 / Q^{2}$ suppression, provided we choose the appropriate initial condition for eq. (103). The contribution of this term to the evolution equation is consequently small. To understand the structure of the result we write the solution of eq. (103) in the region of small $\beta$ ( $\omega \rightarrow 0$ ), where the emission of extra gluons and quarks could be important (as have been seen in section 3). In the region of small $\omega$ we can neglect $\gamma^{-}$(see for example Ref. 40.) and the solution has a simple form

$$
\begin{gathered}
\Sigma_{P}\left(Q^{2}, \omega\right)= \\
\left\{\Sigma_{P}\left(Q_{0}^{2}, \omega\right) \frac{\gamma^{-}(\omega)}{\gamma^{+}(\omega)-\gamma^{-}(\omega)}+G_{P}\left(Q_{0}^{2}, \omega\right) \frac{2 N_{F} P_{G}^{S}(\omega)}{\gamma^{+}(\omega)-\gamma^{-}(\omega)}\right\} e^{\gamma^{+}(\omega) \ln \left(Q^{2} / Q_{0}^{2}\right)} \\
+\int_{Q_{0}^{2}}^{Q^{2}} \frac{d Q^{\prime 2}}{Q^{\prime 4}} \frac{\gamma_{2}(\omega)\left[\Phi_{P ; T}^{S}(\omega)+\Phi_{P}^{G}(\omega)\right]}{\tilde{g}_{2}(\omega)-\gamma^{+}(\omega)}\left(x_{P} G\left(Q^{\prime 2}, x_{P}\right)\right)^{2}
\end{gathered}
$$

where $\tilde{g}_{2}(\omega) \rightarrow \frac{4 N_{c} \alpha_{\mathrm{S}}}{\pi \omega}-1$ at $\omega \rightarrow 0$, and $\Phi_{P}(\omega)$ is the $\omega$ image of the Pomeron splitting functions of eq. (104). They are of the form

$$
\begin{gathered}
\Phi_{P ; T}^{S}=\frac{\alpha_{\mathrm{S}}}{N_{C}}\left\{\frac{1}{\omega+2}-\frac{2}{\omega+3}+\frac{1}{\omega+4}\right\} \\
\Phi_{P ; T}^{G}=\frac{\alpha_{\mathrm{S}} N_{C}^{2}}{2\left(N_{C}^{2}-1\right)}\left\{\frac{1}{\omega}+\frac{2}{\omega+1}-\frac{3}{\omega+2}-\frac{3}{\omega+3}-\frac{4}{\omega+4}+\frac{4}{\omega+5}\right\}
\end{gathered}
$$

To fix the two initial moments $\Sigma_{P}\left(Q_{0}^{2}, \omega\right)$ and $G_{P}\left(Q_{0}^{2}, \omega\right)$ which enter the solution of the phenomenological term $f_{S O F T}^{D D}$ at low $\beta$, we need to choose a phenomenological input. We use Regge phenomenology to fix this term 41] rewriting it in the form

$$
f_{S O F T}^{D D}\left(Q_{0}^{2}, x_{P}, \omega\right)=\left(\frac{1}{x_{P}}\right)^{2 \varepsilon_{s o f t}} f_{S O F T}^{D D}\left(Q_{0}^{2}, \omega\right)
$$

where $F^{D D}\left(Q_{0}^{2}, \omega\right)$ is the $\omega$-image of $G_{P R P} \beta^{\Delta_{R}(0)}(1-\beta)^{n} \cdot \Delta_{R}(0)=\alpha_{R}(0)-1$ where $\alpha_{R}(0)$ is the intercept of the secondary Regge trajectory $\left(\alpha_{R}(0) \sim 0.5\right)$. The power $n$ is not defined in Regge phenomenology and we only know that $n>1$. G $G_{P R P}$ denotes the triple Pomeron-Regge-Pomeron vertex 41]. The second term is the hard diffractive contribution, which is calculated theoretically.

There is an alternate way to implement the initial condition, i.e. to use the results of our calculations in the low mass region. We showed in our calculations for small mass 
production, that the typical virtuality is sufficiently large. Therefore, we can choose the value of the initial virtuality $Q_{0}^{2}$ for the DD process to be about $1-2 \mathrm{GeV}^{2}$ and calculate $f_{S O F T}^{D D}$ in $\mathrm{pQCD}$. In this case

$$
\Sigma_{P}\left(Q_{0}^{2}, \omega\right)=\frac{1}{Q_{0}^{2}} \Phi_{P ; T}^{S}(\omega)\left(x_{P} G\left(Q^{\prime 2}, x_{P}\right)\right)^{2}
$$

and

$$
G_{P}\left(Q_{0}^{2}, \omega\right)=\Phi_{P}^{G}(\omega)\left(x_{P} G\left(Q^{\prime 2}, x_{P}\right)\right)^{2}
$$

This input corresponds to the initial distributions in the $\beta$ - representation

$$
\begin{aligned}
& \Sigma_{P}\left(Q_{0}^{2}, x_{P}, \beta\right)=\frac{1}{Q_{0}^{2}} \Phi_{P ; T}^{S}(\beta)\left(x_{P} G\left(Q_{0}^{2}, x_{P}\right)\right)^{2} \\
& G_{P}\left(Q_{0}^{2}, x_{P}, \beta\right)=\frac{1}{Q_{0}^{2}} \Phi_{P ; T}^{G}(\beta)\left(x_{P} G\left(Q_{0}^{2}, x_{P}\right)\right)^{2}
\end{aligned}
$$

These distributions are plotted in Fig.16 for different values of $x_{P}$ at two $Q_{0}^{2}$ values $\left(Q_{0}^{2}=1 \mathrm{GeV}^{2}\right.$ and $\left.Q_{0}^{2}=2 \mathrm{GeV}^{2}\right)$.

We wish to emphasis two points. First, we propose to seperate between the soft and hard diffraction processes in a way which conforms with the factorization theorem 42 for the integrated diffractive structure function. Although, there is no proof that the factorization theorem holds for the case of diffractive contribution, in DIS 43, all known contributions which violate the factorization theorem turn out to be small 38 444. 45 .

The second point concerns the value of the scale $Q_{0}$. There is no reason to take the same value of $Q_{0}$, both in the evolution equation and in the DIS structure function. Depending on the choice of the scale $Q_{0}^{2}$, we can start the evolution with very small values of $Q_{0}$ and use Regge phenomenology as an initial condition for the DD structure function. Or, we can start with a sufficiently large value of $Q_{0}$ in the DD processes, and reconstruct the initial distributions using pQCD. We followed the second alternative, and our calculations for small masses supports this strategy.

\section{Conclusions}

DD processes in DIS provide a new window to collective phenomena in the parton cascades since they originate from parton-parton interactions and vanish if there is no interaction between the partons. However, DD processes possess an inherent awkwardness in that it is difficult to predict what is small or large in DD. Indeed, in two extreme limits for small 
and for large ("black disc") parton-parton interactions, the cross section of the DD turns out to be small. In this paper an attempt is made to discuss the DD process within a pQCD framework.

Our main results are:

1. The DD cross section originates from small distances $\left(r_{\perp}<0.2 \mathrm{fm}\right)$ even for a transverse polarised photon. This fact justifies our use of pQCD for DD processes.

2. For $\beta>0.4$ only production of $\bar{q} q$ pairs contributes to the cross section of DD. This suggests a method to extract the value of the gluon density from the DD measurements in this kinematic region.

3. For $\beta>0.7$ the longitudinal polarised photon provides the dominant contibution to DD processes. DD of a longitudinal photon is one of the most promising processes for $\mathrm{pQCD}$ calculations, since only small distances $\left(r_{\perp} \propto 1 / Q\right)$ contibute to this process. Therefore, the measurement of DD at such large values of $\beta$ allows one to extract the value of the gluon density. It has all the advantages of vector meson production in DD 125 but without any of the uncertainties due to our poor knowledge of the hadronic wave function [30 46.

4. The general approach to the evolution equations for DD has been formulated and solutions to the evolution equations have been found. The main difference between the evolution equations for DD and the DGLAP evolution equations for the DIS structure functions, is the appearance of the inhomogenious term in the DD equations. The form of this term and its influence on the solution has been discussed. The inhomogenious term in the DD evolution equations (eq. (103)) changes the behaviourof $\frac{\partial \Sigma_{P}}{\partial \ln Q^{2}}$ at large values of $\beta$ adding a positive contribution. This contribution may change our conclusion that the Pomeron gluon density peaks at $\beta \rightarrow 1$, which was based on the DGLAP evolution without the inhomogenious term.

5. The formulae for SC have been obtained and the damping factors have been calculated. The main result is that SC are important in the case of DD, and the value of the cross section for DD processes allows us to estimate the corrections to the DIS structure function which turns out to be fairly large, $\frac{\Delta F_{2}}{F_{2}} \approx 2 \frac{\sigma^{D D}}{\sigma_{t o t}}$.

We consider our paper as a first step in the understanding of DD as a new source of information about collective phenomena in DIS in the region of small $x$. We hope this paper will give some impetus to treat DD as a "hard" process, within the solid theoretical framework of pQCD.

Acknowledgement: E. M. Levin wishes to thank LAFEX-CBPF for their kind hospitality and $\mathrm{CNPq}$ (Brazil) for partial financial support. While completing this paper, our attention was drawn to two recent papers 47] 48] which deal with some of the topics covered in this paper. 


\section{References}

[1] J. Bartels and M. Loewe: Z. Phys. C 12 (1982) 263.

[2] L.V. Gribov, E.M. Levin and M.G. Ryskin: Phys. Rep. 100 (1983) 1; J. Bartels and G. Ingelman: Phys. Lett. B235 (1990) 175; M.G Ryskin: Sov. J. Nucl. Phys. 52 (1990) 529; N.N. Nikolaev and B.G. Zakharov: Z. Phys. C 49 (1991) 607, Phys. Lett. B260 (1991) 414; Z. Phys. C53 (1992) 331; E. Levin and M. Wusthoff: Phys. Rev. D50 (1994) 4306.

[3] M.G. Ryskin: Z. Phys. C57 (1993) 89.

[4] S.J. Brodsky et.al.: Phys. Rev. D50 (1994) 3134.

[5] N.N. Nikolaev and B.G.Zakharov: Z. Phys. C49 (1991) 607; Phys. Lett. B260 (1991) 414.

[6] M.G Ryskin: Sov. J. Nucl. Phys. 52 (1990) 529.

[7] E. Levin and M. Wüsthoff: Phys. Rev. D50 (1994) 4306.

[8] E.M. Levin and M.G.Ryskin: Sov. J. Nucl. Phys. 45 (1987) 1.

[9] A.H. Mueller: Nucl. Phys. B335 (1990) 115; E. Levin and M. Wuesthoff: Phys. Rev. D50 (1994) 4306

[10] E. Gotsman, E.M. Levin and U. Maor: Phys. Lett. B353 (1995) 526

[11] H1 Collaboration, T. Ahmed et.al.: Nucl. Phys. B429 (1994) 477; Phys. Lett. B348 (1995) 681.

[12] ZEUS Collaboration, M. Derrick et.al.: Phys. Lett. B315 (1993) 481; B332 (1994) 228; B338 (1994) 483.

[13] ZEUS collaboration, G. Wolf: "Rapidity Gaps in DIS ( ZEUS )" talk at Eilat Conference on Diffractive Scattering, February 1996, Eilat, Israel; ZEUS collaboration, M. Derrick et.al.: DESY 96-018, February 1996. 
[14] H1 collaboration, Andrew Mehta: "Rapidity gaps in DIS (H1)" talk at Eilat Conference on Diffractive Scattering, February 1996, Eilat, Israel.

[15] M. Gluck, E. Reya and A. Vogt: Z. Phys. C53 (1992) 127.

[16] V.N. Gribov and L.N. Lipatov: Sov. J. Nucl. Phys. 15 (1972) 438; L.N. Lipatov: Yad. Fiz. 20 (1974) 181; G. Altarelli and G. Parisi: Nucl. Phys. B 126 (1977) 298; Yu.L.Dokshitzer: Sov.Phys. JETP 46 (1977) 641.

[17] E.A. Kuraev, L.N. Lipatov and V.S. Fadin: Sov. Phys. JETP 45 (1977) 199; Ya.Ya. Balitskii and L.V. Lipatov: Sov. J. Nucl. Phys. 28 (1978) 822; L.N. Lipatov: Sov. Phys. JETP 63 (1986) 904.

[18] T. Jaroszewicz: Phys. Lett. B116 (1982) 291

[19] A.H. Mueller: Nucl. Phys. B415 (1994) 373

[20] L. V. Gribov, L.V. Gribov, E. M. Levin and M. G. Ryskin: Phys.Rep. 100 (1983) 1.

[21] M. Abramowitz and I.A. Stegun: Handbook of Mathematical Functions, Dover Publications, INC, NY 1970.

[22] B.Z. Kopeliovich et.al.: Phys. Lett. B324 (1994) 469.

[23] H. Abramowicz, L. Frankfurt and M. Strikman: DESY-95-047, March 1995.

[24] A.H.Mueller and J. Qiu: Nucl. Phys. B268 (1986) 427.

[25] E. Gotsman, E. Levin and U. Maor: Nucl. Phys. B464 (1996) 251.

[26] V.A. Abramovski,V.N. Gribov and O.V. Kancheli: Sov. J. Nucl. Phys. 18 (1973) 308

[27] E.M. Levin and M.G. Ryskin: Phys.Rep. 189 (1990) 267.

[28] E.M. Levin and M.G. Ryskin: Sov. J. Nucl. Phys. 50 (1989) 881; Z. Phys. C48 (1990) 231

[29] E. Gotsman, E.M. Levin and U. Maor: Phys. Lett. B309 (1993) 109; Z. Phys. C57 (1993) 667; Phys. Rev. D49 (1994) R4321; Phys. Lett. B347 (1995) 424

[30] L. Frankfurt, W. Koepf and M. Strikman: TAUP-2290/95,hep-ph/9509311.

[31] A.D. Martin, R.G. Roberts and W.J. Stirling: Phys. Lett. B354 (1995) 155. 
[32] A.Donnachie and P.V.Landshoff: Nucl. Phys. B244 (1984) 322; Nucl. Phys. B276 (1986) 690; Nucl. Phys. B231 (1989) 189; Phys. Lett. B296 (1992) 227.

[33] H.L. Lai et.al., CTEQ collaboration: Phys. Rev. D51 (1995) 4763

[34] G. Ingelman and P.Sclein: Phys. Lett. B152 (1985) 256

[35] ZEUS Collaboration, M. Derrick et.al.: Phys. Lett. B356 (1995) 601; H1 Collaboration, S. Aid et.al.: DESY 96-023, February 1996.

[36] H1 collaboration, G. Knies: "Aligned jet analysis of DIS rapidity gap events in H1" talk at Eilat Conference on Diffractive Scattering, February 1996, Eilat, Israel.

[37] Yu.L. Dokshitzer, V.A. Khoze and S.I. Troyan: Z. Phys. C55 (1992) 107

[38] J. Bartels: Z. Phys. C60 (1993) 471; Phys. Lett. B298 (1993) 204; E.M. Levin, M.G. Ryskin and A.G. Shuvaev: Nucl. Phys. B387 (1992) 589

[39] Yu.L. Dokshitser et.al.: "Basis of perturbative QCD", ed. Edition Frontier.

[40] R.K. Ellis, Z. Kunszt and E.M. Levin: Nucl. Phys. B420 (1994) 517

[41] A. Capella et.al.: Phys. Lett. B345 (1995) 403; K. Golec-Biernat and J. Kwiecinski: Phys. Lett. B353 (1995) 329

[42] J. Collins, D.E. Soper and G. Sterman: Nucl. Phys. B308 (1988) 833; In "Perturbative Quantum Chromodynamics", ed. A.H. Mueller, Singapore, WS 1989 and reference therein.

[43] J. Collins, L. Frankfurt and M. Strikman: Phys. Lett. B307 (1993) 161

[44] E. Levin: "Deep Inelastic Scattering and Related Subjects" Eilat, Israel, February 1994, ed. A. Levy, WS 1994, p. 83; A. Berrera and D. Soper: Phys. Rev. D50 (1994) 4328

[45] J. Bartels and M. Wüsthoff: Z. Phys. C66 (1995) 157

[46] M.G. Ryskin, R.G. Roberts, A.D. Martin and E.M. Levin: DTP-95-96, hepph/9511228.

[47] M. Genovese, N.N.Nikolaev and B.G. Zakharov: KFA-IKP(Th) 1996-1, hepph/9602246.

[48] J. Bartels, H. Lotter and M. Wüsthoff: DESY 96-026, hep-ph/9602362. 


\section{Figure Captions.}

Fig.1: Diffractive production of a quark-antiquark pair.

Fig.2: Extra gluon emission in diffractive production.

Fig.3: The damping factor $\left(D_{L}^{2}\right)$ for a longitudinal polarised photon.

Fig.4: $\quad$ The damping factor $\left(D_{T}^{2}\right)$ for a transverse polarised photon.

Fig.5: $\quad$ The damping factor $\left(D_{e l}^{2}\right)$ for $F_{2}\left(Q^{2}, x\right)$.

Fig.6: DD by longitudinal and transverse polarised photons at $\beta=0.8$.

Fig.7: $\quad$ The integrand of eq. (28).

Fig.8: $\quad x_{P} F_{2}^{D(3)}$ versus $x_{P}$, calculations and HERA data.

Fig.9: $\quad$ Effective power $n\left(Q^{2}, \beta\right)$ and the ratio $\frac{x_{P} G\left(Q^{2}, x_{P}\right)^{L O}}{x_{P} G\left(Q^{2}, x_{P}\right)^{N L O}}$ in the GRV paramerization.

Fig.10: Different contributions to $F_{2}^{D D(2)}$.

Fig.11: Comparison of $F_{2}^{D(2)}$ with the HERA experimental data.

Fig.12: The ratio R (eq. (80)) for different values of $k_{0}$.

Fig.13: The transverse momentum distribution for the DD jet.

Fig.14: The cross section for $\rho$ production (in arbitrary units) for eqs. (82) and (83).

Fig.15: The initial parton distribution for the DD evolution equations with longitudinal polarised photons.

Fig.16: The initial parton distributions for the DD evolution equations with transverse polarised photons. 


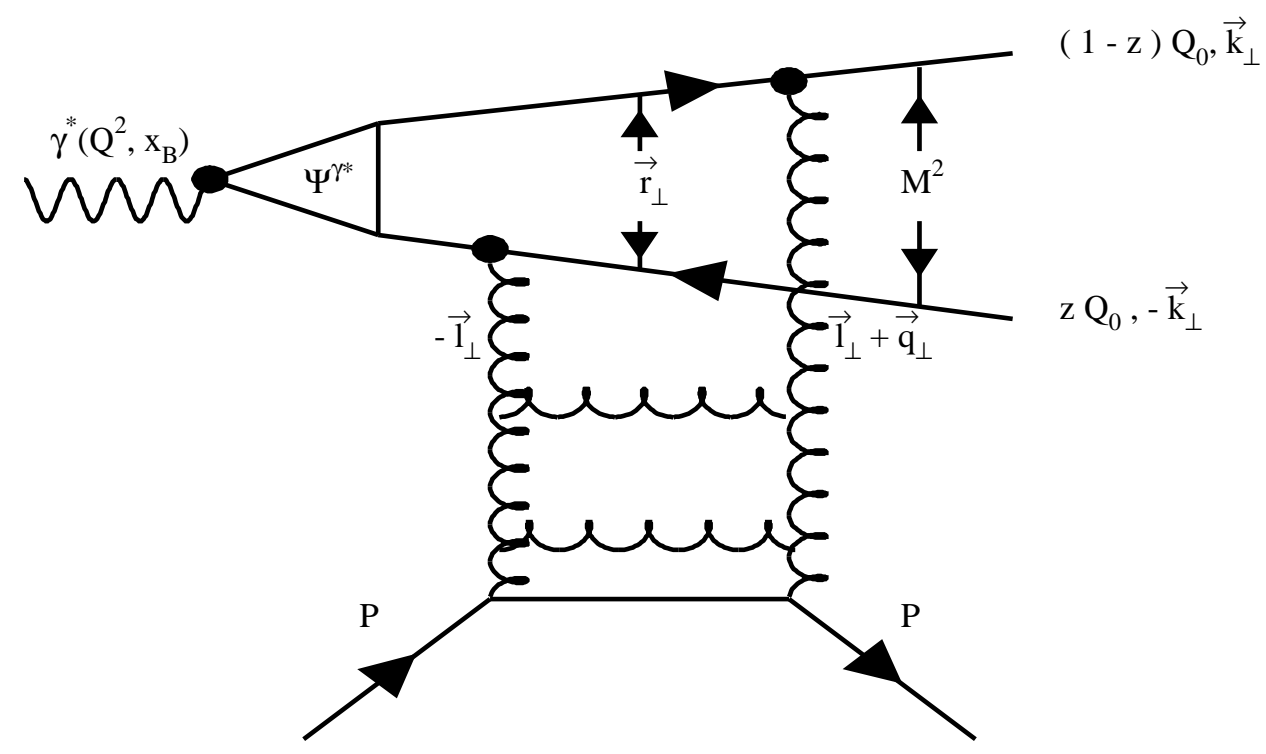

Figure 1: The diffractive production of quark - antiquark pair. 

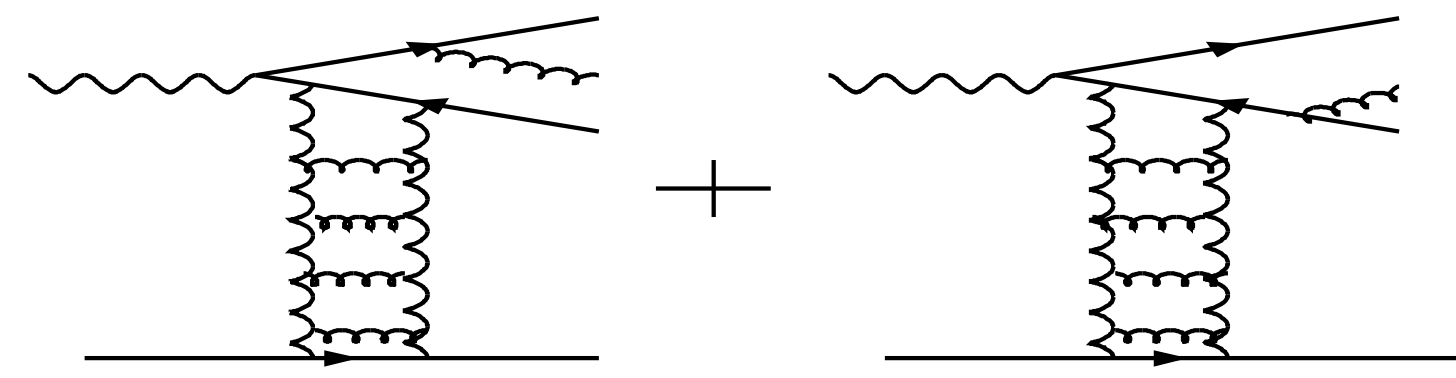

Fig. 2a

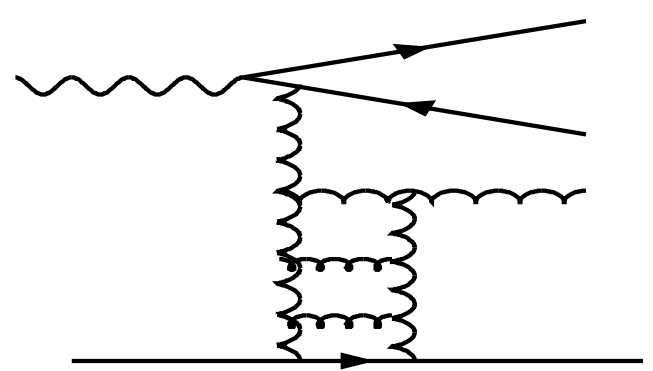

Fig. $2 b$

Figure 2: The extra gluon emission in the diffractive production. 


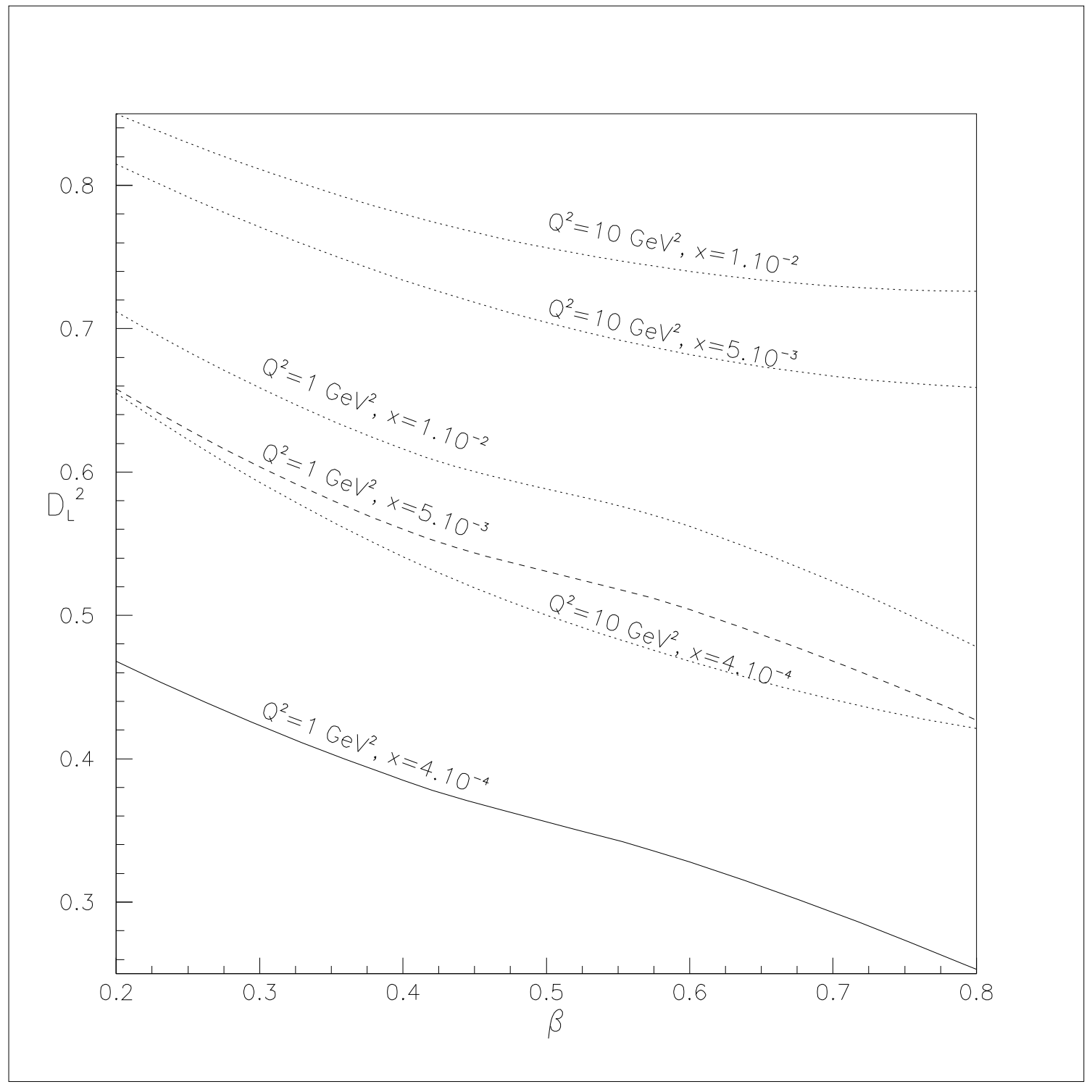

Figure 3: The damping factor for longitudinally polarised photon. 

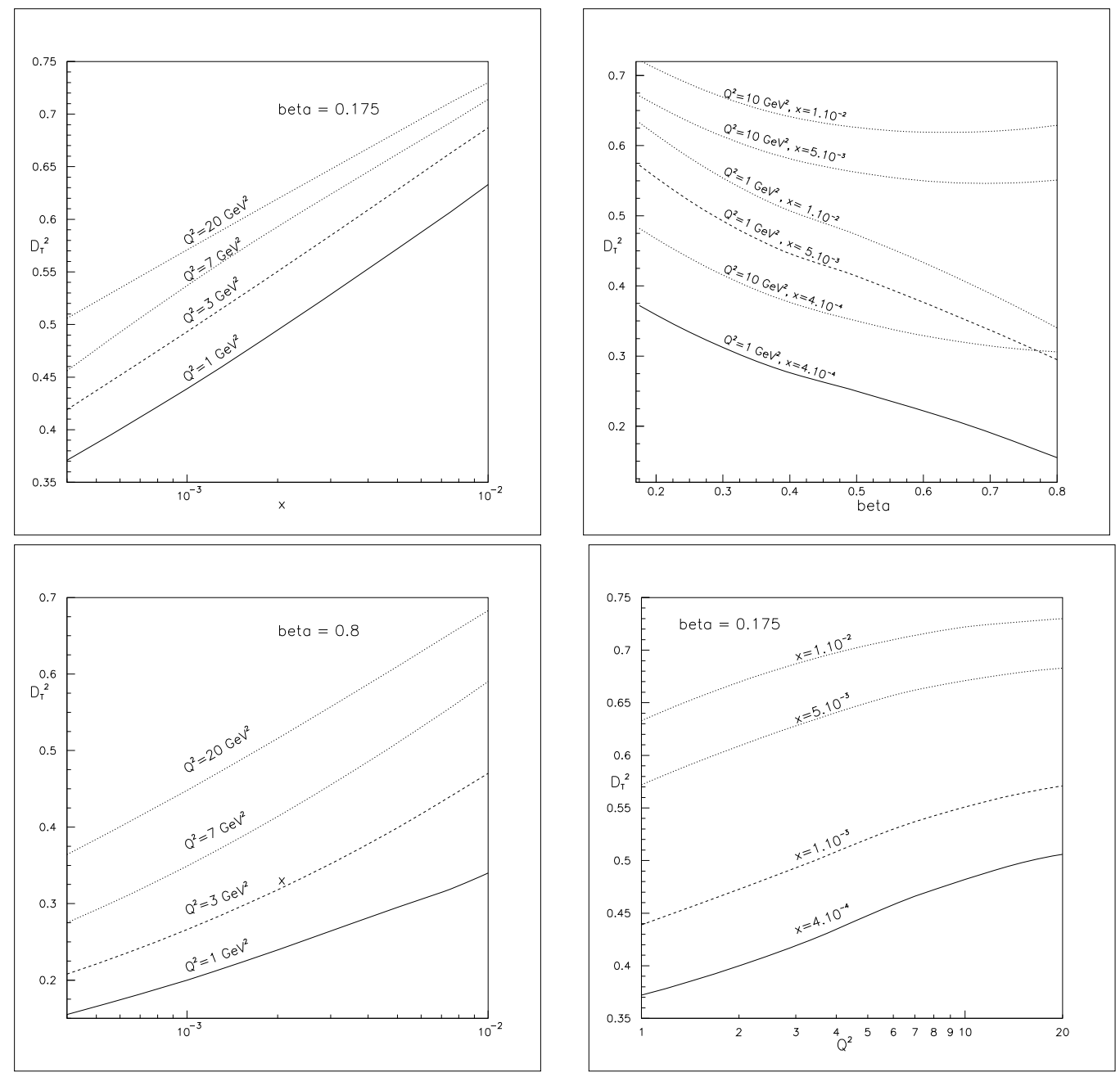

Figure 4: The damping factor for the transversely polarised photon. 


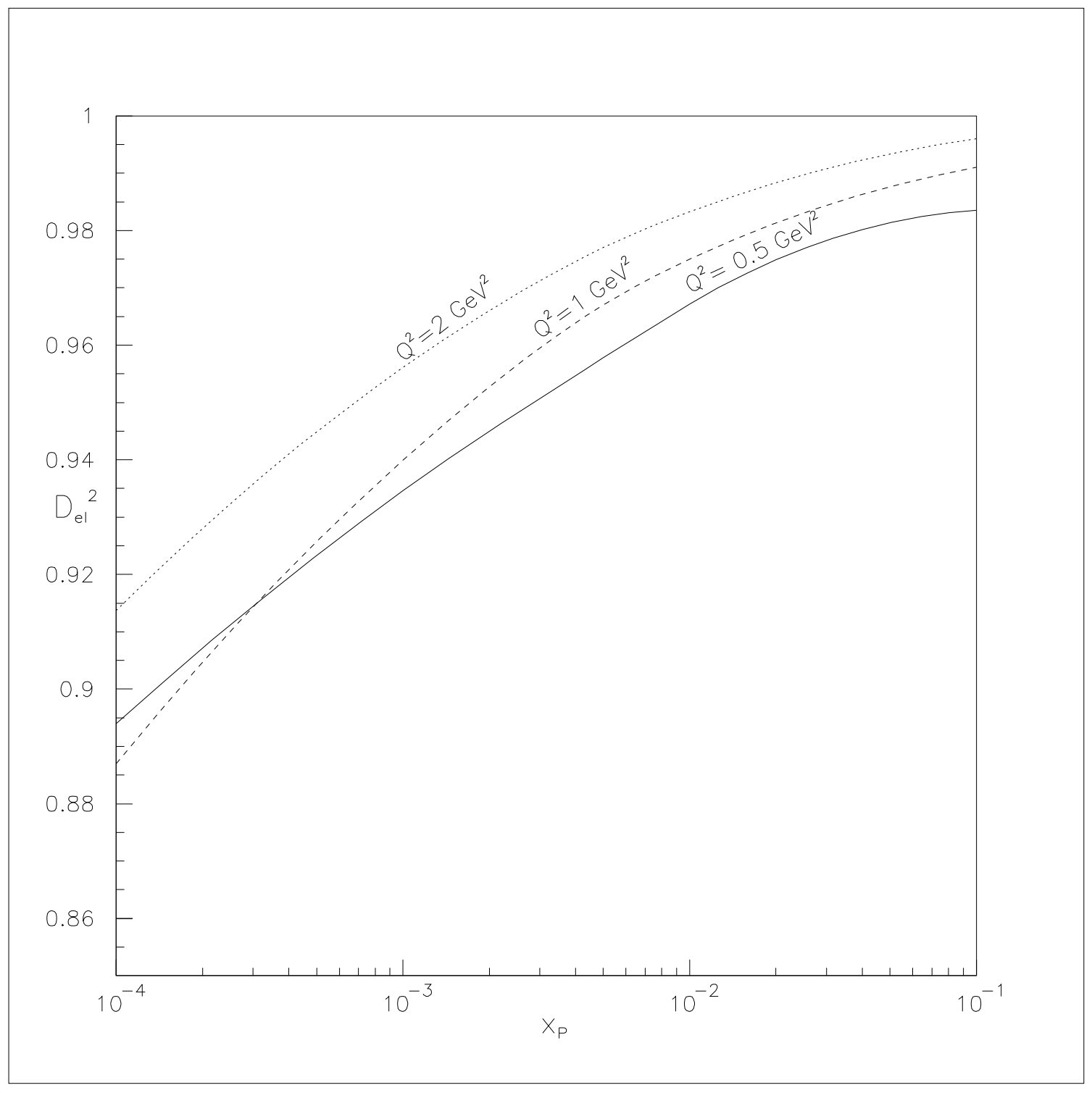

Figure 5: The damping factor $\left(D_{e l}^{2}\right)$ for $F_{2}\left(x, Q^{2}\right)$. 


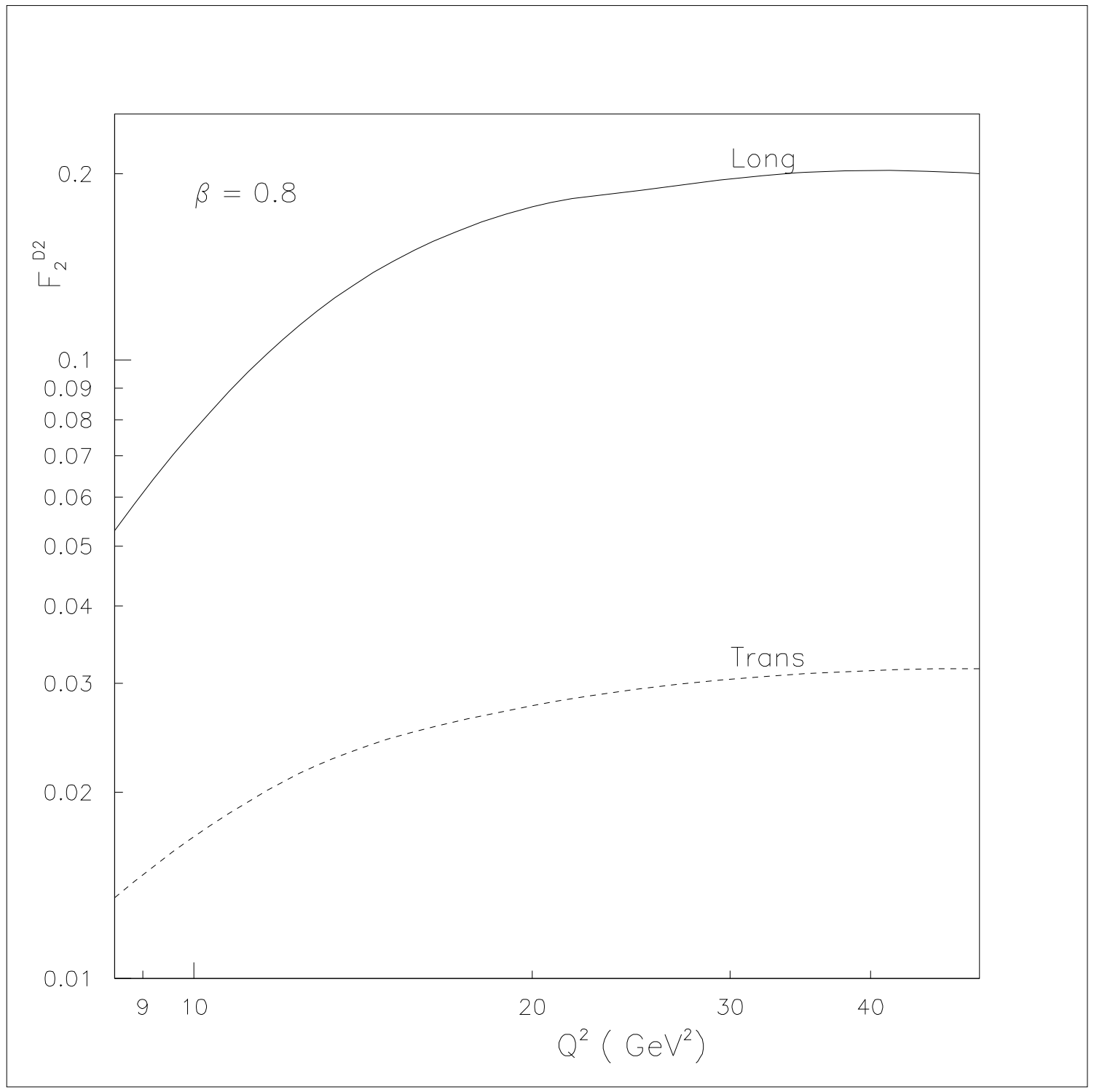

Figure 6: The DD by longitudinally and transversely polarised photon at $\beta$ $=0.8$. 


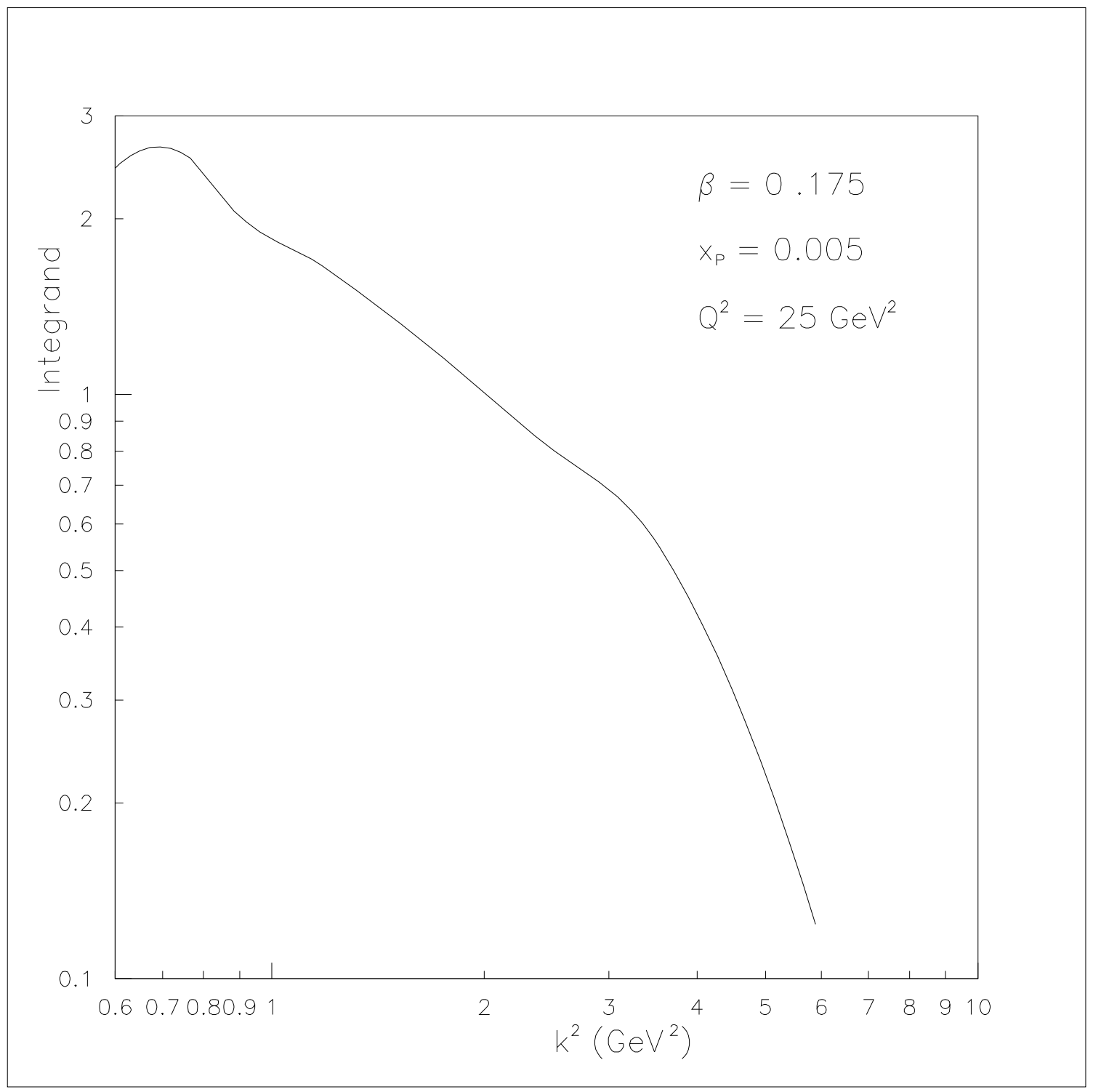

Figure 7: The integrand of Eq.(28). 

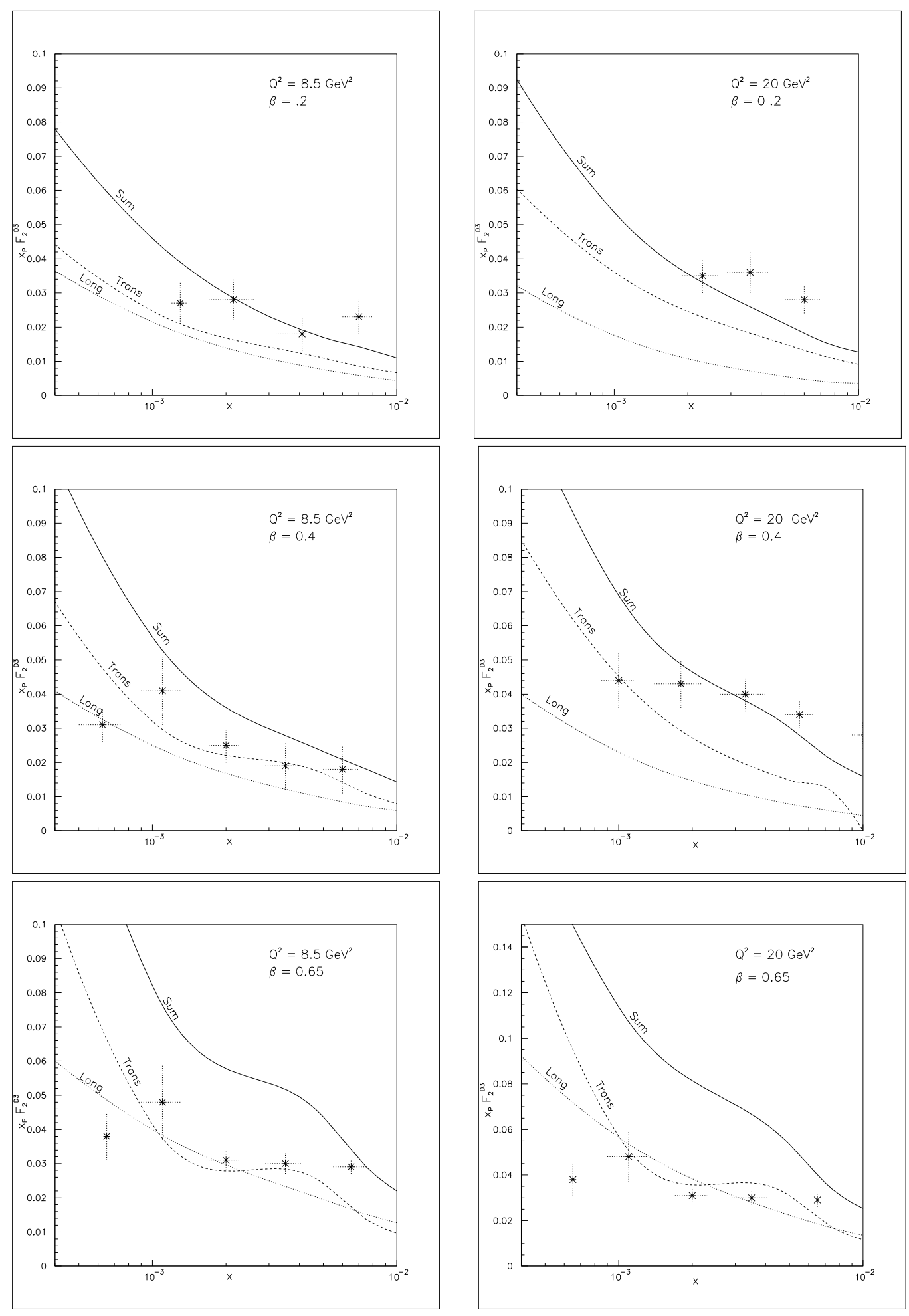

Figure 8: $x_{P} F_{2}^{D D(3)}$ versus $x_{P}$. 

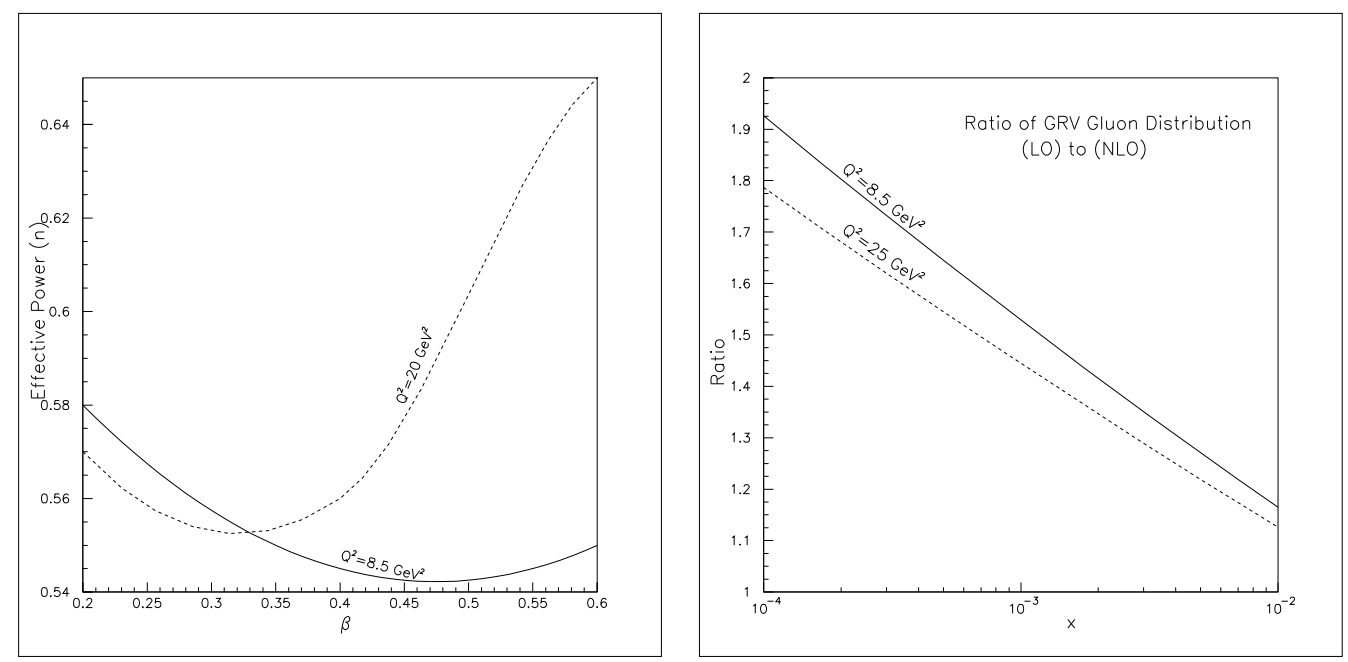

Figure 9: Effective power $n\left(\underline{,} Q^{2}\right)$ and ratio $\frac{x G\left(x, Q^{2}\right)^{L O}}{x G\left(x, Q^{2}\right)^{N L O}}$ in the GRV parametrization. 

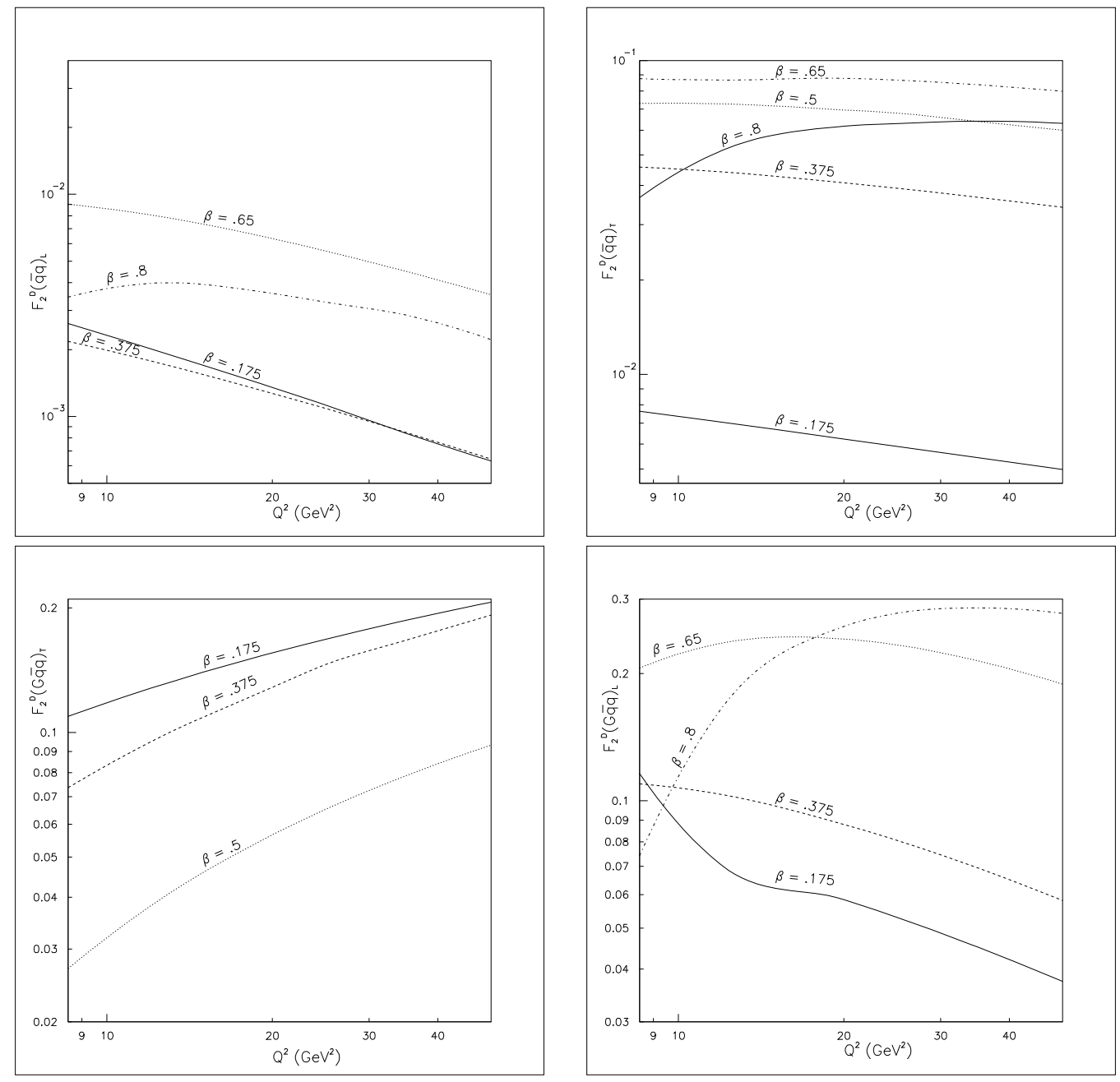

Figure 10: Different contributions to $F_{2}^{D D(2)}$. 

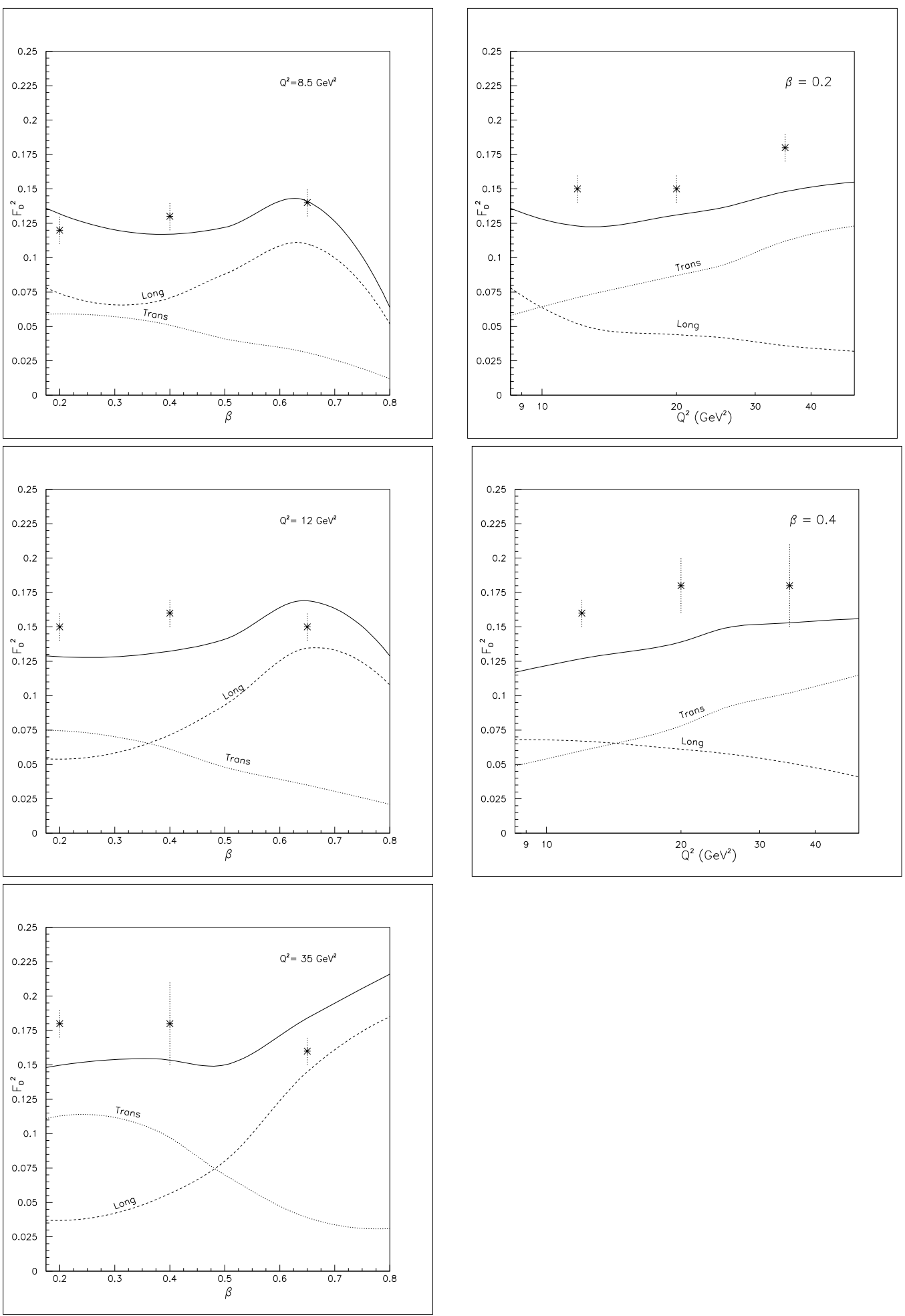

Figure 11: Comparison of $F_{2}^{D D(2)}$ with the HERA experimental data. 


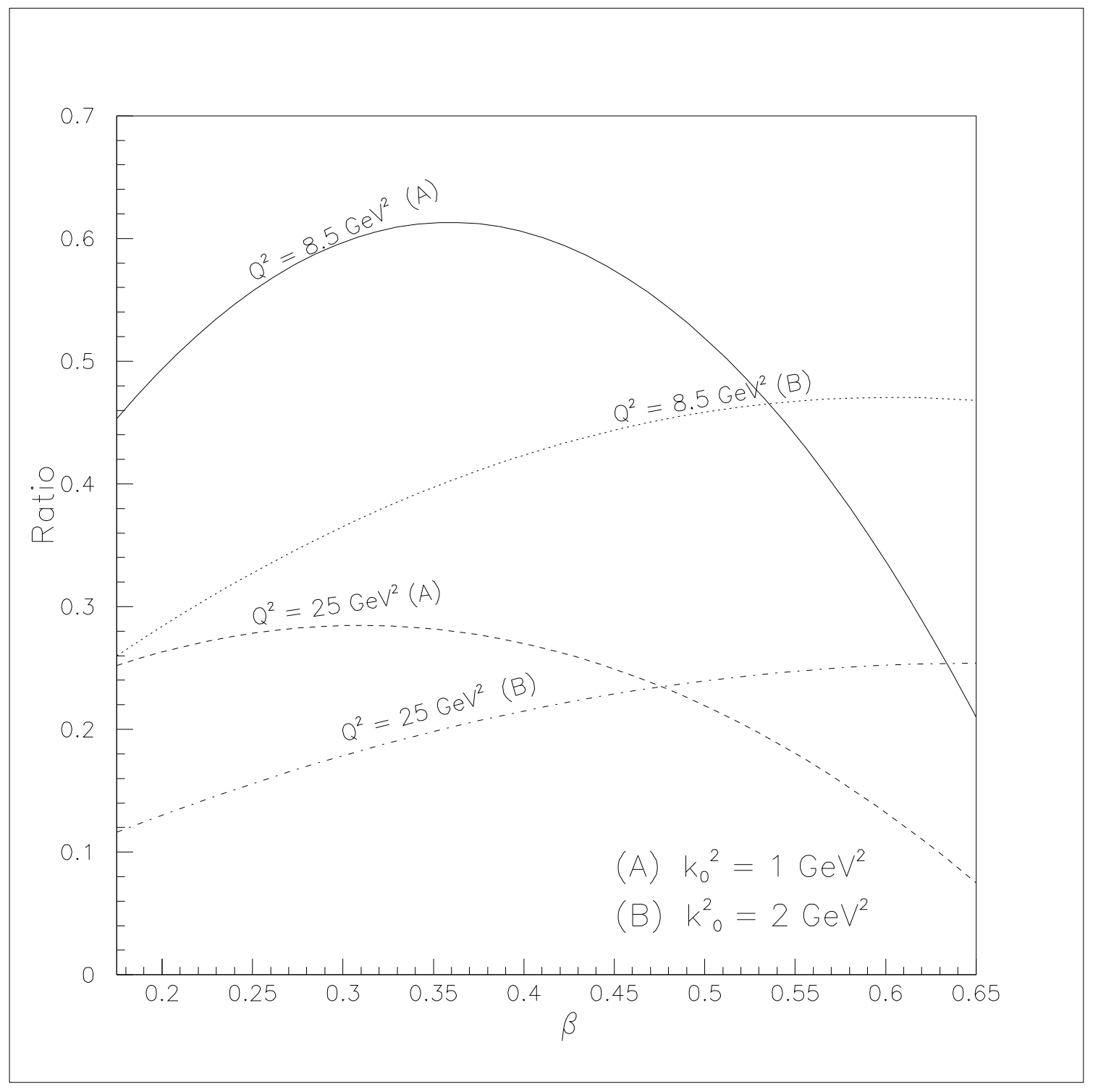

Figure 12: Ratio R (Eq.(75)) for different values of $k_{0}$. 


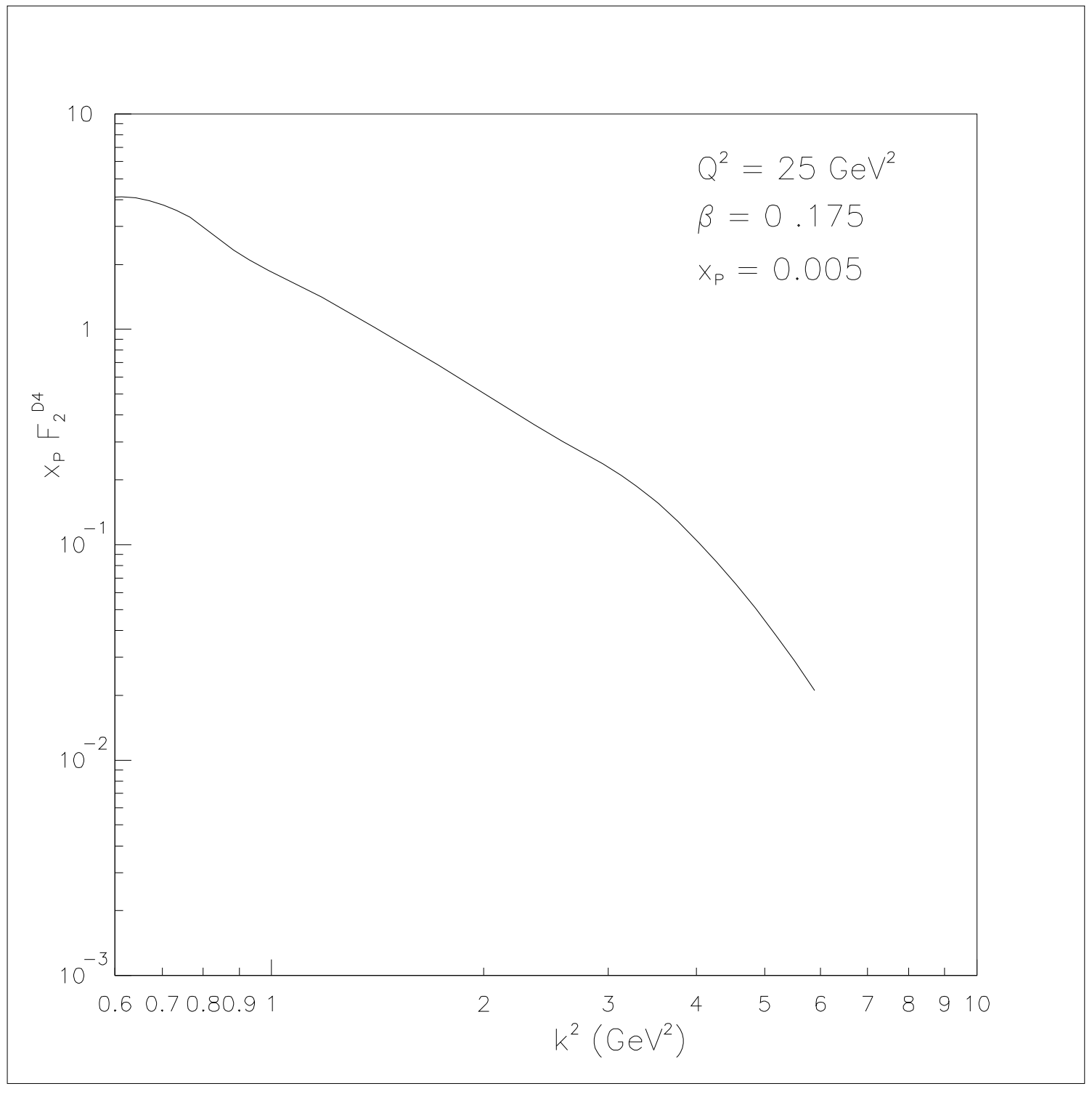

Figure 13: The transverse momentum distribution for the jet in the DD. 


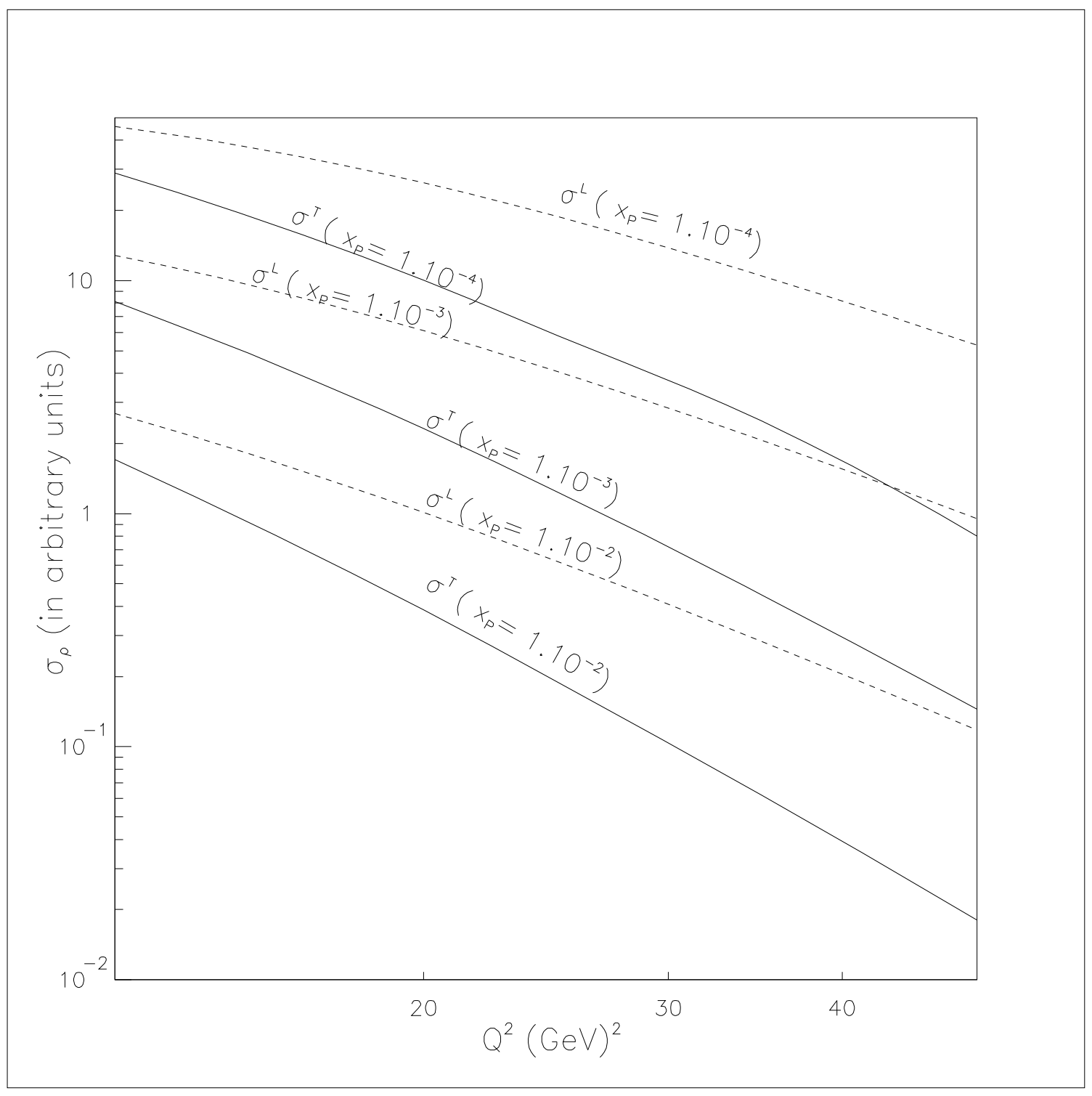

Figure 14: The cross section for $\rho$ production (in arbitrary units) for Eqs.(77) and (78). 


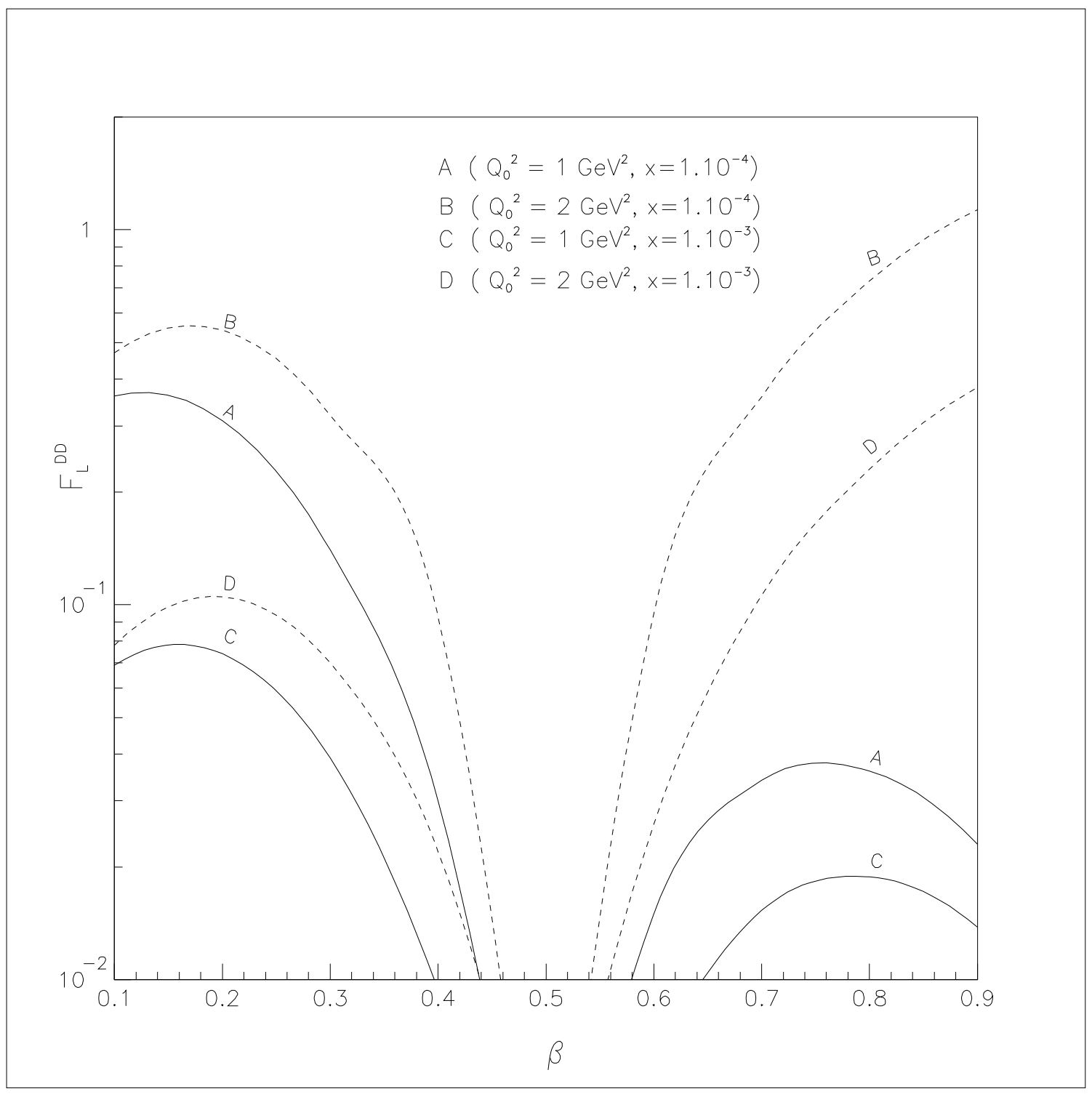

Figure 15: The initial quark distribution for the evolution equation for longitudinally polarised photon. 

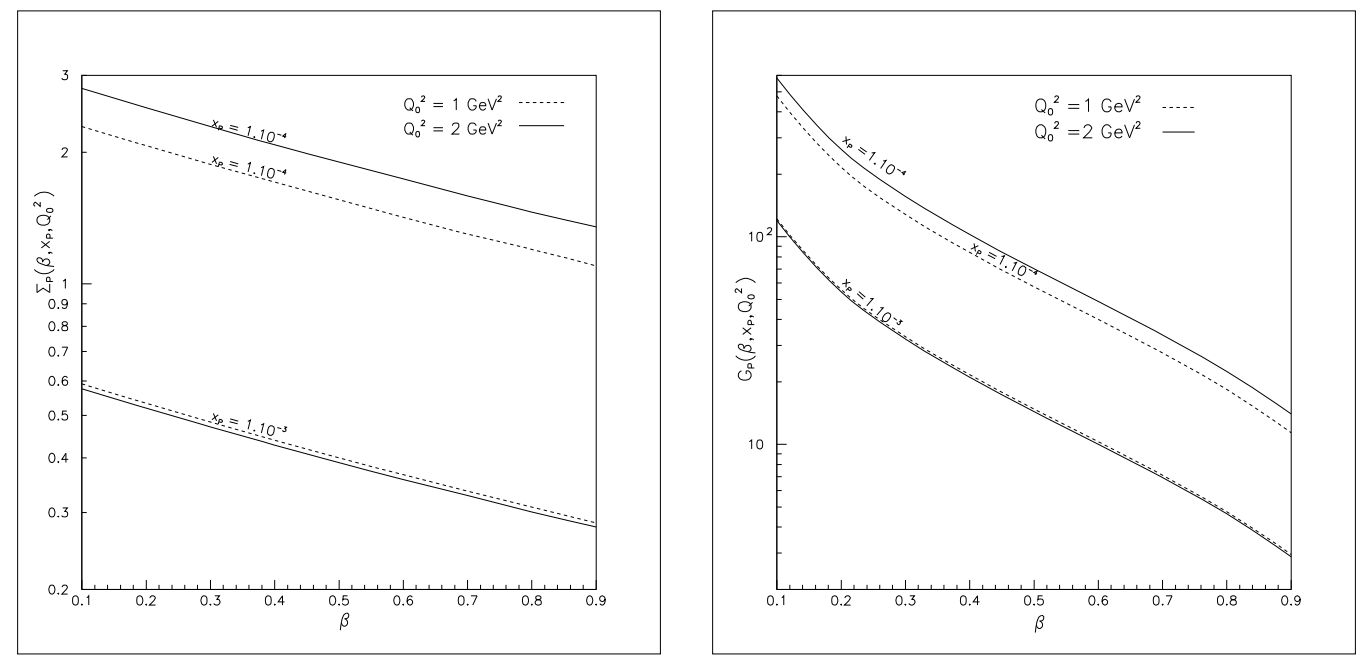

Figure 16: The initial quark distribution for the evolution equation for transverselly polarised photon. 